\title{
Attractors for Delayed, Non-Rotational von Karman Plates with Applications to Flow-Structure Interactions Without any Damping
}

\author{
Igor Chueshov \\ Kharkov National University \\ Kharkov, UA \\ chueshov@univer.kharkov.ua \\ Irena Lasiecka \\ University of Virginia \\ Charlottesville, VA \\ il2v@virginia.edu
}

October 20, 2018

\author{
Justin T. Webster \\ Oregon State University \\ Corvallis, OR \\ websterj@math.oregonstate.edu
}

\begin{abstract}
This paper is devoted to a long time behavior analysis associated with flow structure interactions at subsonic and supersonic velocities. It turns out that an intrinsic component of that analysis is the study of attracting sets corresponding to von Karman plate equations with delayed terms and without rotational terms. The presence of delay terms in the dynamical system leads to the loss of gradient structure while the absence of rotational terms in von Karman plates leads to the loss of compactness of the orbits. Both these features make the analysis of long time behavior rather subtle rendering the established tools in the theory of PDE dynamical systems not applicable. It is our goal to develop methodology that is capable of handling this class of problems.
\end{abstract}

Key terms: nonlinear plate, PDE with delay, long-time behavior of solutions, dynamical systems, global attractors, flow-structure interaction,

MSC 2010: 35L20, 74F10, 35Q74.

\section{Introduction}

The study of von Karman plates in the presence of aerodynamical forces represented by some delayed functional is physically motivated [3, 20, 22, These models with delay often arise in the modeling of coupled dynamics (e.g., fluid or flow-structure interaction) where the impact of the off-plate dynamics can be written as a boundary value of some delayed (flow) potential in the plate equation after a sufficiently large time. In fact, this is the case for flow-plate interactions arising in the modeling of panels and plates immersed in an inviscid flow (for some discussion, see [6] and [14]), and thus we can reduce the study of long-time behavior of solutions to the coupled flow-plate system to the problem of a von Karman plate forced by some delay term.

In the present work, the motivation and significance of studying this class of models derives from recent developments in the area of flow-structure interactions, with the goal of attaining good mathematical understanding of flow-structure dynamics at both subsonic and supersonic flow velocities. It is 
known from experiment (and also confirmed by numerics), that the potential flow (particularly at the supersonic speeds) has the ability of inducing a certain amount of stability in the moving structure. This is the case even when the structure itself does not possess mechanical damping mechanisms. If one writes down the equations for the interactive system, along with the standard energy balance, this dissipative effect is not exhibited at all; quantities are conserved and not dissipated. Thus, there must be some "hidden" mechanism which produces this dissipation. It is our goal to shed some light on this phenomenon. As it turns out, the decoupling technique introduced in [5, 6], which reduces the analysis of full flow-structure interaction to that of a certain delayed plate model, allows us to observe certain stabilizing effects of the flow. These occur in the form of non-conservative forces acting upon the structure as the "downwash" of the flow. This idea was already applied to Berger plate models [7, 17] in the proof of existence of attractors corresponding to the associated reduced plate problem with a delayed term.

In fact, well-posedness and long-time behavior analyses of nonlinear plate PDEs with delays have been treated in [8] (see also [14): first, in the case of the von Karman model with rotational inertia, and secondly, in [7, 17, in the case of the Berger model with a small intensity of delayed term (this corresponds to a large speed $U$ of the flow of gas - hypersonic). These expositions flesh out the existence and properties of global attractors for the general plate with delay in the presence of a 'natural' form of interior damping, and then apply this general result to the specific delayed (aeroelastic) force given in the full flow-plate coupling.

It should be noted that the presence of rotational inertia parameter, while drastically improving the topological properties of the model, is neither natural nor desirable in the context of flow-structure interaction. First, the original model for flow structure interaction describes the interaction between the mid-surface of the plate and flow above the plate; and hence should the plate should be treated two-dimensionally so the equation describing the "downwash" of the flow and the oscillations of the plate can agree on the interface. Thus, the rotational inertia term (proportional to the cube of the plate thickness, see, e.g., [29]) should be neglected. Secondly, the presence of rotational term changes the kinetic energy in the system, forcing a much stronger stabilization mechanism (abstractly, this corresponds to the well-known fact that the essential spectrum of an operator can not be moved with a compact perturbation). In that case, the stability induced by the flow (a viscous, velocity-proportional damping) does not suffice providing a stabilizing effect on the structure. Instead, when the rotational inertia term is neglected, the damping secured by the flow alone provides the main mechanism for stabilization. This would seem to corroborate physical findings. In view of this, it is paramount to the problem at hand to consider the model which does not account for rotational inertia and imposes no limiting regimes on the flow velocity parameter $U \geq 0, U \neq 1$.

It is the purpose of this paper to show that the requirement of the rotational term and restrictions on the values of $U$ can be eliminated. This is accomplished by resorting to modern analytical tools, including compensated compactness methods. These tools, developed over the last few years [14, are capable of revealing a mathematical structure of the problem which is consistent with numerical and experimental findings. Thus, not only the results, but also the techniques and approach utilized in this paper appear to be significant to the discussion of flow-plate interactions and aeroelasticity. Accordingly, in this treatment, we focus on the more difficult, and hereto open, non-rotational von Karman case, without any restrictions on value of unperturbed speed $(0 \leq U<+\infty, U \neq 1)$.

The mathematical difficulties which arise in this model force us to consider new long-time behavior 
technologies applied within this framework. In order to provide a glimpse of this, and to demonstrate the timeliness of the project undertaken, it suffices to note that the first (and most fundamental) difficulty one is faced with is the well-posedness of finite energy solutions corresponding to flowstructure interactions - in particularly in the supersonic case. Results and methods found in past literature depend critically on the presence of rotational inertial term, see [5, 6] and also [14, Chapters 6 and 12]. Only recently has this problem has been solved for the non-rotational model for all flow velocities [16] (the subsonic case was also discussed earlier in [4, 36] and also in [14). Equipped with dynamical system structure for the flow-plate interaction, one may then proceed with a study of long time behavior.

The main points of this treatment are: (i) to give a unified approach to the long-time behavior of this model, beginning with well-posedness discussions, and working to the existence and finitedimensionality of a global attractor. Our results are novel, and they complete the analysis of long-time behavior of von Karman systems with delay by addressing the non-rotational case and the associated resulting lack of compactness. (ii) We make use of a recent technique [11, 12, 14, 15, 24] which allows us to obtain the asymptotic compactness property for the dynamical system without making use of any gradient type structure of the dynamics (not available in this model, owing to the dispersive flow term). This has the added benefit of producing extra regularity of the attractor, and is a less demanding approach (the 'traditional' approach of showing quasistability (on the attractor) for von Karman plates requires a full characterization of the attractor or assumptions on finiteness of the stationary points of the dynamical system and the use of backward-in-time methods, which are not available in systems with delay). Lastly, (iii) we make use of new breakthroughs (both in well-posedness and hidden compactness) with regard to von Karman flow-plate interactions in order to apply our general results on this system with delay to an aeroelasticity model which has received considerable attention from the PDE and dynamical systems community, as well as the engineering and aeroscience community, over the past 20 years. The results following from the application are also novel, and provide a complete treatment of the long-time behavior of a von Karman full flow-plate interaction model. Namely, for this model we show the existence of a finite-dimensional compact set in the phase space of the plate component. This set attracts all plate trajectories in the case when the corresponding gas flow initial data are localized. We emphasize that this result does not assume any damping imposed on the system. While stability of flow structure interactions without any damping has been experienced both numerically and experimentally, our works appears to be a first rigorous mathematical treatment of this phenomena. The key ingredients of the analysis include: exhibit of "hidden" dissipation related to the dispersive character of the flow equation along with "hidden" regularity of the boundary traces of the flow, the decoupling technique introduced in [6] and applied within the context of recent and powerful techniques aimed at showing asymptotic smoothness and quasi-stability [14 without the a-priori known compactness or gradient structure.

We also note that stability and flutter control in flow structure interactions has been also treated by several authors at different levels (see, e.g., 2, 3, 21, 23, and the references therein). For instance the recent monograph 2] contains a detailed derivation of the flow equation coupled to a linear beam equation with the boundary data which involve the Kutta-Jukovsky circulation condition for the flow and hence can be applied to elastic wings dynamics. The main trust of the corresponding analysis (see also 33 and the literature cited therein) is the characterization of unstable aeroelastic modes arising in Possio equation which is linear integral equation describing aerodynamic pressure on the structure. 
The associated spectral analysis is focused on finding unstable modes in the linear dynamics. The obtained results [2, 33] provide specific information on the flutter speed. In contrast, our results show that long time behavior of a nonlinear flow-plate model can be reduced to a finite dimensional attracting set. More precise information on the stability/unstability of finite dimensional orbits would require in depth study of the resulting nonlinear finite dimensional dynamical system (which can be chaotic in the supersonic case).

The paper is organized as follows. In Section 2 we describe the general flow-plate interaction models and its relation to the model with delay. We also state well-posedness results here (Proposition 2.10) and sketch a proof. This result allows us to define a corresponding evolution semigroup. In Section 3 we state and discuss our main result on long-time dynamics of the delayed model (Theorem 3.1). We also show what consequences this result yields for long-time dynamics of the general flow-plate interaction model (Theorem 3.3). The next section, Section 4 is central and devoted to the proofs of the main results. In Section 5 we briefly discuss the proof of the reduction theorem (Theorem 2.3) which rigorously ties the full flow-plate dynamics to the evolution of the von Karman plate with delay. Finally, in the Appendix we establish some needed properties of the delayed (aerodynamic type) force.

Notation: For the remainder of the text we write $\mathbf{x}$ for $(x, y, z) \in \mathbb{R}_{+}^{3}$ or $(x, y) \in \Omega \subset \mathbb{R}_{\{(x, y)\}}^{2}$, as dictated by context. Norms $\|\cdot\|$ are taken to be $L_{2}(D)$ for the domain dictated by context. Inner products in $L_{2}\left(\mathbb{R}_{+}^{3}\right)$ are written $(\cdot, \cdot)$, while inner products in $L_{2}\left(\mathbb{R}^{2} \equiv \partial \mathbb{R}_{+}^{3}\right)$ are written $<\cdot, \cdot>$. Also, $H^{s}(D)$ will denote the Sobolev space of order $s$, defined on a domain $D$, and $H_{0}^{s}(D)$ denotes the closure of $C_{0}^{\infty}(D)$ in the $H^{s}(D)$ norm which we denote by $\|\cdot\|_{H^{s}(D)}$ or $\|\cdot\|_{s, D}$. We make use of the standard notation for the trace of functions defined on $\mathbb{R}_{+}^{3}$, i.e. for $\phi \in H^{1}\left(\mathbb{R}_{+}^{3}\right), \gamma[\phi]=\left.\phi\right|_{z=0}$ is the trace of $\phi$ on the plane $\{\mathrm{x}: z=0\}$.

\section{Motivation and Description of the Model}

\subsection{Flow-Plate Interactions}

The model we begin with describes the interaction between a nonlinear plate with a field or flow of gas above it. To describe the behavior of the gas we make use of the theory of potential flows (see, e.g., 3, 20, 27, and the references therein) which produces a perturbed wave equation for the velocity potential of the flow. The oscillatory behavior of the plate is governed by the second order (in time) Kirchhoff plate equation with a general nonlinearity. We consider the von Karman nonlinearity, which is used in the modeling of the large oscillations of thin, flexible plates - so-called large deflection theory. These equations are well known in nonlinear elasticity and constitute a basic model describing nonlinear oscillations of a plate accounting for large displacements, see [25] and also [14, 18, 29] (and references therein).

The gas flow environment we consider is $\mathbb{R}_{+}^{3}=\{(x, y, z): z>0\}$. The plate is immersed in an inviscid flow (over body, $z \leq 0$ ) with velocity $U \neq 1$ in the negative $x$-direction. (Here we normalize $U=1$ to be Mach 1, i.e. $0 \leq U<1$ is subsonic and $U>1$ is supersonic.) This situation, for instance, corresponds to the dynamics of a panel element of an aircraft flying with the speed $U$, see, e.g., 21, 22.

The plate is modeled by a bounded domain $\Omega \subset \mathbb{R}_{\{(x, y)\}}^{2}=\{(x, y, z): z=0\}$ with smooth boundary $\partial \Omega=\Gamma$ and the scalar function $u: \Omega \times \mathbb{R}_{+} \rightarrow \mathbb{R}$ represents the vertical displacement of the plate in 
the $z$-direction at the point $(x, y)$ at the moment $t$. We focus on the plate with clamped boundary condition: 1 .

Accepting von Karman large deflection hypotheses we arrive at the following system:

$$
\left\{\begin{array}{l}
u_{t t}+\Delta^{2} u+k u_{t}+f(u)=p(\mathbf{x}, t) \quad \text { in } \Omega \times(0, T), \\
u=\partial_{\nu} u=0 \quad \text { on } \partial \Omega \times(0, T), \\
u(0)=u_{0}, \quad u_{t}(0)=u_{1} .
\end{array}\right.
$$

We take the nonlinearity to be von Karman:

$$
f(u)=-\left[u, v(u)+F_{0}\right],
$$

where $F_{0}$ is a given forcing function and the von Karman bracket $[u, v]$ is given by

$$
[u, v]=\partial_{x}^{2} u \partial_{y}^{2} v+\partial_{y}^{2} u \partial_{x}^{2} v-2 \partial_{x y}^{2} u \partial_{x y}^{2} v
$$

and the Airy stress function $v(u)$ is defined by the relation $v(u)=v(u, u)$, where $v(u, w)$ solves the following elliptic problem

$$
\Delta^{2} v(u, w)+[u, w]=0 \quad \text { in } \Omega, \quad \partial_{\nu} v(u, w)=v(u, w)=0 \text { on } \partial \Omega,
$$

for given $u, w \in H_{0}^{2}(\Omega)$.

For the flow component of the model, we make use of linearized potential theory, and we know [3, 21] that the (perturbed) flow potential $\phi: \mathbb{R}_{+}^{3} \rightarrow \mathbb{R}$ must satisfy the perturbed wave equation below (note that when $U=0$ this is the standard wave equation):

$$
\begin{cases}\left(\partial_{t}+U \partial_{x}\right)^{2} \phi=\Delta \phi & \text { in } \mathbb{R}_{+}^{3} \times(0, T), \\ \phi(0)=\phi_{0} ; \quad \phi_{t}(0)=\phi_{1}, & \\ \partial_{\nu} \phi=d(\mathbf{x}, t) & \text { on } \mathbb{R}_{\{(x, y)\}}^{2} \times(0, T) .\end{cases}
$$

The strong coupling here takes place in the downwash term of the flow potential (the Neumann boundary condition) by taking

$$
d(\mathbf{x}, t)=-\left[\left(\partial_{t}+U \partial_{x}\right) u(\mathbf{x})\right] \cdot \mathbf{1}_{\Omega}(\mathbf{x}), \quad \mathbf{x} \in \mathbb{R}^{2},
$$

and by taking in (2.1) the aerodynamical pressure of the form

$$
p(\mathbf{x}, t)=p_{0}(\mathbf{x})+\left(\partial_{t}+U \partial_{x}\right) \gamma[\phi] \quad \text { with }\left.\gamma[\phi] \equiv \phi\right|_{z=0} .
$$

Here above $\mathbf{1}_{\Omega}(\mathbf{x})$ denotes the indicator function of $\Omega$ in $\mathbb{R}^{2}$. This structure of $d(\mathbf{x}, t)$ corresponds to the case when the part of boundary $z=0$ outside of the plate is a the surface of a rigid body.

\footnotetext{
1 The clamped boundary conditions are the most physically relevant boundary conditions for the flow-plate model; additionally, clamped boundary conditions allow us to avoid certain technical issues in the consideration and streamline our exposition. Other possible and relevant plate boundary conditions in this setup include: free, hinged (or simply supported), hinged dissipation, and combinations thereof [14, 29] 30].
} 
This gives the fully coupled model:

$$
\begin{cases}u_{t t}+\Delta^{2} u+k u_{t}+f(u)=p_{0}+\left(\partial_{t}+U \partial_{x}\right) \gamma[\phi] & \text { in } \Omega \times(0, T), \\ u(0)=u_{0} ; \quad u_{t}(0)=u_{1}, & \\ u=\partial_{\nu} u=0 & \text { on } \partial \Omega \times(0, T), \\ \left(\partial_{t}+U \partial_{x}\right)^{2} \phi=\Delta \phi & \text { in } \mathbb{R}_{+}^{3} \times(0, T), \\ \phi(0)=\phi_{0} ; \quad \phi_{t}(0)=\phi_{1}, & \text { on } \mathbb{R}_{\{(x, y)\}}^{2} \times(0, T) .\end{cases}
$$

In this situation, a complete description of well-posedness would require an in depth discussion of strong solutions to the (2.6) system, including the semigroup formulation and discussion of the generator of the dynamics. In addition, these results are not uniform with respect to the parameter value $U$. We refer the reader interested in these details to [4, 13, 16, 36, see also [14] and the references therein. For this treatment, the key point is the well-posedness of weak solutions to (2.6). These weak solutions satisfy the variational formulation as defined in [14. For the purpose of this work we simply cite a recently obtained [16] well-posedness result which attests that the dynamical system generated by (2.6) is associated to a strongly continuous semigroup on the phase space

$$
H \equiv H_{0}^{2}(\Omega) \times L_{2}(\Omega) \times H^{1}\left(\mathbb{R}_{+}^{3}\right) \times L_{2}\left(\mathbb{R}_{+}^{3}\right)
$$

The corresponding result proved in [16] is stated below.

Theorem 2.1. Let $0 \leq U \neq 1, k \geq 0$ and $F_{0} \in H^{3+\delta}(\Omega), p_{0} \in L_{2}(\Omega)$. With reference to the system defined in (2.6) and any initial data $y_{0} \equiv\left(u_{0}, u_{1} ; \phi_{0}, \phi_{1}\right) \in H$, there exists a unique solution $y(\cdot) \in C([0, \infty) ; H)$ which is represented by a strongly continuous semigroup $T_{t}: H \rightarrow H, y(t)=T_{t} y_{0}$, $t>0$, with the estimate

$$
\left\|T_{t} y_{0}-T_{t} z_{0}\right\|_{H} \leq C(R) e^{\omega_{R} t}\|y(0)-z(0)\|_{H}, \quad \forall\left\|y_{0}\right\|_{H} \leq R,\left\|z_{0}\right\|_{H} \leq R
$$

where $C(R)$ and $\omega_{R}$ are positive constants.

Remark 2.2. When $0 \leq U<1$ the semigroup $T_{t}$ in Theorem 2.1 is stable in some extended space $\widetilde{H}$, i.e. there is a space $\widetilde{\widetilde{H}} \supset H$ such that $\left\|T_{t} y\right\|_{\widetilde{H}} \leq C(R), t>0$. The above estimate (valid also for $k=0$ ) owes its validity to the nonlinear effects [36, 13, 14. It is not valid for the corresponding linear semigroup.

Various past considerations (se, e.g., [14]) of systems like (2.6) have made use of an explicit solver for the flow. In such an approach, we may rewrite the system above as a von Karman system with delay of the form in the earlier sections. Reducing the flow-plate problem to a delayed von Karman plate is the primary motivation for this treatment and allows long-time behavior analysis of the flow-plate system, which is considerably more difficult otherwise. The exact statement of this reduction is given in the following assertion:

Theorem 2.3. Let the hypotheses of Theorem 2.1 be in force, and $\left(u_{0}, u_{1} ; \phi_{0}, \phi_{1}\right) \in H_{0}^{2}(\Omega) \times L_{2}(\Omega) \times$ $H^{1}\left(\mathbb{R}_{+}^{3}\right) \times L_{2}\left(\mathbb{R}_{+}^{3}\right)$. Assume that there exists an $R$ such that $\phi_{0}(\mathbf{x})=\phi_{1}(\mathbf{x})=0$ for $|\mathbf{x}|>R$. Then the 
there exists a time $t^{\#}(R, U, \Omega)>0$ such that for all $t>t^{\#}$ the weak solution $u(t)$ to (2.6) satisfies the following equation:

$$
u_{t t}+\Delta^{2} u+k u_{t}-\left[u, v(u)+F_{0}\right]=p_{0}-\left(\partial_{t}+U \partial_{x}\right) u-q^{u}(t)
$$

with

$$
q^{u}(t)=\frac{1}{2 \pi} \int_{0}^{t^{*}} d s \int_{0}^{2 \pi} d \theta\left[M_{\theta}^{2} \widehat{u}\right](x-(U+\sin \theta) s, y-s \cos \theta, t-s) .
$$

Here, $\widehat{u}$ is the extension 2 of $u$ by 0 outside of $\Omega ; M_{\theta}=\sin \theta \partial_{x}+\cos \theta \partial_{y}$ and

$$
t^{*}=\inf \{t: \mathbf{x}(U, \theta, s) \notin \Omega \text { for all } \mathbf{x} \in \Omega, \theta \in[0,2 \pi], \text { and } s>t\}
$$

with $\mathbf{x}(U, \theta, s)=(x-(U+\sin \theta) s, y-s \cos \theta) \subset \mathbb{R}^{2}$.

Thus, after some time, the behavior of the flow can be captured by the aerodynamical pressure term $p(t)$ in the form of a reduced delayed forcing. This representation has been used in previous considerations of long-time behavior of plates and shallow shells [14, Section 6.6, pp. 312-334] (and the references therein). A rigorous proof of this representation can be found in [14, pp. 333-334] for the rotational case (when we have additional regularity of the plate velocity $u_{t}$ ). For the reader's convenience, in Section 5 we provide a sketch of the proof, which extends the arguments given in 14 for the rotational case. This extension is direct, once the following ingredients are accounted for: (1) The full system in (2.6) generates strongly continuous semigroup (see Theorem 2.11), (2) the von Karman bracket is locally Lipschitz on $H$ (see Lemma 2.11), and (3) the time derivative of the delayed term $q_{t}^{u}$ is bounded on $H$ (inequality (2.13) in Proposition 2.4) .

Theorem 2.3 allows us to suppress the dependence of the problem on the flow variable $\phi$. Here we emphasize that the structure of aerodynamical pressure (2.5) posited in the hypotheses leads to the velocity term $-u_{t}$ on the RHS of (2.7). One can be absorb this term into the damping coefficient $k$ on the LHS. However, since we have made no assumptions on the value of $k$, we may strengthen our result for the full reduced flow-plate system by henceforth assuming $k=0$ and utilizing the natural damping appearing in the structure of the reduced flow pressure, i.e., by moving this term to the RHS.

As we see below, the reduction method above allows us to study long-time behavior of the dynamical system corresponding to (2.6) (for sufficiently large times) by reducing the problem to a plate equation with delay. The flow state variables $\left(\phi, \phi_{t}\right)$ manifest themselves in our rewritten system via the delayed character of the problem; they appear in the initial data for the delayed component of the plate, namely $\left.u^{t}\right|_{\left(-t^{*}, 0\right)}$. Hence the behavior of both dynamical systems agree for all $t>t(R, U, \Omega)$. By the dynamical systems property for the full system (see Theorem 2.1), we can propagate forward and simply study the long-time behavior of the plate with delay on the interval $\left(\sigma-t^{*}, \sigma+T\right]$ for $\sigma>t_{\#}$ and $T \leq \infty$.

The following proposition motivates the hypotheses imposed below on the delayed force term in the von Karman plate model (2.7).

Proposition 2.4. Let $q^{u}(t)$ be given by (2.8). Then

$$
\left\|q^{u}(t)\right\|_{-1}^{2} \leq c t^{*} \int_{t-t^{*}}^{t}\|u(\tau)\|_{1}^{2} d \tau
$$

\footnotetext{
${ }^{2}$ This extension of the solution $u(t)$ is possible owing to the clamped boundary conditions.
} 
for any $u \in L_{2}\left(t-t^{*}, t ; H_{0}^{1}(\Omega)\right)$. If $u \in L_{2}^{\text {loc }}\left(\left[-t^{*},+\infty\left[; H^{2} \cap H_{0}^{1}\right)(\Omega)\right)\right.$ we also have

$$
\left\|q^{u}(t)\right\|^{2} \leq c t^{*} \int_{t-t^{*}}^{t}\|u(\tau)\|_{2}^{2} d \tau, \quad \forall t \geq 0
$$

and

$$
\int_{0}^{t}\left\|q^{u}(\tau)\right\|^{2} d \tau \leq c\left[t^{*}\right]^{2} \int_{-t^{*}}^{t}\|u(\tau)\|_{2}^{2} d \tau, \quad \forall t \geq 0 .
$$

Moreover if $\left.u \in C\left(-t^{*},+\infty ; H^{2} \cap H_{0}^{1}\right)(\Omega)\right)$, we have that $q^{u}(t) \in C^{1}\left(\mathbb{R}_{+} ; H^{-1}(\Omega)\right)$,

$$
\left\|q_{t}^{u}(t)\right\|_{-1} \leq C\left\{\|u(t)\|_{1}+\left\|u\left(t-t^{*}\right)\right\|_{1}+\int_{-t^{*}}^{0}\|u(t+\tau)\|_{2} d \tau\right\}, \quad \forall t \geq 0 .
$$

For the proof, we refer to Section 6 below.

Remark 2.5. A priori, when $u_{t}$ is in $H_{0}^{1}(\Omega)$, it is clear from (2.10) that there is a compactness margin and we have the estimate

$$
\int_{0}^{t}<q^{u}(\tau), u_{t}(\tau)>d \tau \leq \epsilon \int_{0}^{t}\left\|u_{t}(\tau)\right\|_{1}^{2}+C(\epsilon, t) \sup _{\tau \in\left[-t^{*}, t\right]}\|u(\tau)\|_{1}^{2} .
$$

However, this is not immediately apparent when $u_{t} \in L_{2}(\Omega)$ as $\left\|q^{u}(t)\right\|_{0}^{2}$ has no such a priori bound from above, as in (2.10). Hence, the critical component which allows us a transition from the $\gamma>0$ case (with damping of the form $k(1-\gamma \Delta) u_{t}$ ) to the $\gamma=0$ case is the hidden compactness of the aforementioned term displayed by (2.13). We note that inequality (2.13) represents a loss of one derivative (anisotropic - time derivatives are scaled by two spatial derivatives), versus the loss of two derivatives in (2.10), (2.11), and (2.12).

\subsection{PDE Description of the Plate Model with the Delay}

Below we utilize a positive parameter $0<t^{*}<+\infty$ as the time of delay, and accept the commonly used (see, e.g., [19] or [35) notation $u^{t}(\cdot)$ for function on $s \in\left[-t^{*}, 0\right]$ of the form $s \mapsto u(t+s)$. We need this because of the delayed character of the problem which requires initial data of the prehistory interval $\left[-t^{*}, 0\right]$, i.e., need to impose an initial condition of the form $\left.u\right|_{t \in\left(-t^{*}, 0\right)}=\eta(\mathbf{x}, t)$, where $\eta$ is a given function on $\Omega \times\left[-t^{*}, 0\right]$. We can choose this prehistory data $\eta$ in different classes. In our problem it is convenient to deal with Hilbert type structures, and therefore we assume in the further considerations that $\eta \in L_{2}\left(-t^{*}, 0 ; H_{0}^{2}(\Omega)\right)$. Since we do not assume the continuity of $\eta$ in $s \in\left[-t^{*}, 0\right]$, we also need to add the (standard) initial conditions of the form $u(t=0)=u_{0}(\mathbf{x})$ and $\partial_{t} u(t=0)=u_{1}(x)$.

Again, employing von Karman large deflection hypotheses we arrive at the following system:

$$
\left\{\begin{array}{l}
u_{t t}+\Delta^{2} u+k u_{t}+f(u)+L u=p_{0}+q\left(u^{t}, t\right) \quad \text { in } \Omega \times(0, T), \\
u=\partial_{\nu} u=0 \quad \text { on } \partial \Omega \times(0, T), \\
u(0)=u_{0}, \quad u_{t}(0)=u_{1} \\
\left.u\right|_{t \in\left(-t^{*}, 0\right)}=\eta \in L_{2}\left(-t^{*}, 0 ; H_{0}^{2}(\Omega)\right) .
\end{array}\right.
$$

Here $f(u)$ is given by (2.2). The forcing term $q\left(u^{t}, t\right)$ occurring on the RHS of the plate equation will 
encompass the delayed potential of the gas flow and given by the function $q: L_{2}\left(-t^{*}, 0 ; H_{0}^{2}(\Omega)\right) \times \mathbb{R} \mapsto$ $\mathbb{R}$, which will be specified below. The scalar $k \geq 0$ is our damping coefficient, and represents constant viscous damping across the full interior of the plate. The operator $L$ encompasses spatial lower order terms which do not have gradient structure (e.g., the term $-U u_{x}$ in (2.7)).

Remark 2.6. As it was already mentioned above, the basic plate model we consider may include a rotational inertia term (see, e.g. [29] or [14]), corresponding to the parameter $\gamma \geq 0$ and accompanying damping parameters $k_{1}, k_{2}>0$. This leads to a plate equation of the form

$$
(1-\gamma \Delta) u_{t t}+\Delta^{2} u+\left(k_{1}-k_{2} \Delta\right) u_{t}+f(u)+L u=p_{0}+q\left(u^{t}, t\right) .
$$

Recall that the parameter $\gamma$ corresponds to rotational inertia in the filaments of the plate, as discussed in the Introduction. These kind of delay models were studied in and [8] and [14, Sections 3.3.1 and 9.3.1]. We also note that in the case when

$$
f(u)=f_{0}\left(\int_{\Omega}|\nabla u(\mathbf{x})|^{2} d \mathbf{x}\right)
$$

in (2.14), with an appropriate $C^{1}$ function $f_{0}$, we arrive to the Berger plate model with delay which was studied in [7, 17].

Now we formulate our standing hypotheses; we begin with those responsible for well-posedness of the model in (2.14):

Assumption 2.7. - We suppose $f(u)=-\left[u, v(u)+F_{0}\right]$, where the functions $F_{0}$ and $p_{0}$ possess the properties:

$$
F_{0}(\mathbf{x}) \in H^{3+\delta}(\Omega) \text { for some } \delta>0, \quad p_{0}(\mathbf{x}) \in L_{2}(\Omega) .
$$

- The linear operator $L: H_{0}^{2}(\Omega) \rightarrow L_{2}(\Omega)$ is continuous.

- $v \mapsto q(v, t)$ is a continuous linear mapping from $L_{2}\left(-t^{*}, 0 ; H^{2}(\Omega)\right) \times \mathbb{R}_{+}$to $L_{2}(\Omega)$ possessing the property:

$$
\left\|q\left(u^{t}, t\right)\right\|^{2} \leq C \int_{t-t^{*}}^{t}\|u(\tau)\|_{2}^{2} d \tau, \quad \forall t \geq 0, \quad \forall u \in L_{2}^{l o c}\left(\left[-t^{*},+\infty\left[; H^{2}(\Omega)\right) .\right.\right.
$$

Additional hypotheses are needed for long-time dynamics:

Assumption 2.8. - The linear operator $L: H_{0}^{2-\delta}(\Omega) \rightarrow L_{2}(\Omega)$ is continuous for some $\delta>0$.

- $q(v, t)$ possesses the (additional) property:

$$
\left\|q\left(u^{t}, t\right)\right\|_{-\sigma}^{2} \leq C \int_{t-t^{*}}^{t}\|u(\tau)\|_{2-\sigma}^{2} d \tau \quad \text { for some } 0<\sigma<2
$$

with any $t>0$ and $u \in L_{2}^{\text {loc }}\left(\left[-t^{*},+\infty\left[; H^{2}(\Omega)\right)\right.\right.$.

- We assume that the generalized time derivative $\partial_{t}\left[q\left(u^{t}, t\right)\right]$ belongs to $H^{-2}(\Omega)$ a.s. for any $u \in$ 
$C\left(-t^{*}, T ; H_{0}^{2}(\Omega)\right)$ with the following estimate holding for any $\psi \in H_{0}^{2}(\Omega)$ :

$$
\left|<\partial_{t}\left[q\left(u^{t}, t\right)\right], \psi>\right| \leq C\left(\|u(t)\|_{2}+\left\|u\left(t-t^{*}\right)\right\|_{2}+\int_{-t^{*}}^{0}\|u(t+\tau)\|_{2} d \tau\right)\|\psi\|_{2-\eta}
$$

for some $\eta>0$.

Remark 2.9. By Proposition 2.4 $q\left(u^{t}, t\right) \equiv q^{u}(t)$ given by (2.8) satisfies both Assumptions 2.7 and 2.8. Roughly speaking, the conditions in (2.15) (2.17) mean that the delay time of the system is distributed in the interval $\left[-t^{*}, 0\right]$, with density which is absolutely continuous with respect to Lebesgue measure. This observation also implies that a delay term of the form

$$
q(v, t)=\int_{-t^{*}}^{0} Q(t, \tau) v(\tau) d \tau
$$

where $Q(t, \tau)$ is a family of linear operators from $H_{0}^{2}(\Omega)$ into $L_{2}(\Omega)$ satisfying appropriate hypotheses, could be studies from the point of view of this treatment. We also note that we will use the estimate in (2.17) to derive a result on 'hidden' compactness of the term

$$
\int_{0}^{t}<q\left(u^{\tau}, \tau\right), u_{t}(\tau)>d \tau
$$

which is arrived at via integration by parts in time.

\subsection{Well-Posedness of the Plate Model and Energy Relation}

Long-time behavior analysis of the delayed system depends on the well-posedness of suitably defined weak solutions which generate a dynamical system on the phase space $\mathbf{H}=H_{0}^{2}(\Omega) \times L_{2}(\Omega) \times$ $L_{2}\left(-t^{*}, 0 ; H_{0}^{2}(\Omega)\right)$.

Well-posedness of weak solutions has been addressed [8] and [14, Section 3.3.1, pp. 189-192; 221$222]$ via the Galerkin method, see also [7, 17] in the case of Berger plates. In what follows we summarize and complement relevant results.

We take a weak solution to (2.14) on $[0, T]$ to be a function

$$
u \in L_{\infty}\left(0, T ; H_{0}^{2}(\Omega)\right) \cap W_{\infty}^{1}\left(0, T ; L_{2}(\Omega)\right) \cap L_{2}\left(-t^{*}, 0 ; H_{0}^{2}(\Omega)\right)
$$

such that the variational relation corresponding to (2.14) holds (see, e.g., [14 (4.1.39), p.211]). We now assert:

Proposition 2.10. Let Assumptions 2.7 be in force. Then, with initial data

$$
\left(u_{0}, u_{1}, \eta\right) \in \mathbf{H} \equiv H_{0}^{2}(\Omega) \times L_{2}(\Omega) \times L_{2}\left(-t^{*}, 0 ; H_{0}^{2}(\Omega)\right),
$$

problem (2.14) has a unique weak solution on $[0, T]$ for any $T>0$. This solution belongs to the class

$$
C\left(0, T ; H_{0}^{2}(\Omega)\right) \cap C^{1}\left(0, T ; L_{2}(\Omega)\right)
$$


and satisfies the energy identity

$$
\mathscr{E}(t)+k \int_{s}^{t}\left\|u_{t}(\tau)\right\|^{2} d \tau=\mathscr{E}(s)+\int_{s}^{t}<q\left(u^{\tau}, \tau\right), u_{t}(\tau)>d \tau+\int_{s}^{t}<p_{0}-L u(\tau), u_{t}(\tau)>d \tau,
$$

where the full (not necessarily positive) energy has the form

$$
\mathscr{E}\left(u, u_{t}\right) \equiv \frac{1}{2}\left\{\left\|u_{t}\right\|^{2}-<\left[u, F_{0}\right], u>\right\}+\Pi_{*}(u)
$$

with

$$
\Pi_{*}(u) \equiv \frac{1}{2}\left\{\|\Delta u\|^{2}+\frac{1}{2}\|\Delta v(u)\|^{2}\right\} .
$$

As stated in [14, Section 4.1.6, p.221], the proof of Proposition 2.10 requires only minor modifications with respect to the proof of the related result in Theorem 3.1.1 [14] on p.190. Since Theorem 3.1.1 deals with rotational models $(\gamma>0)$, in order to handle the effect of nonlinear term we rely on the sharp regularity of Airy's stress function $v(u)$, given below:

Lemma 2.11. The function $v(u, w)$ defined in (2.3) satisfies

1. $|v(u, w)|_{W^{2, \infty}(\Omega)} \leq C\|u\|_{2}\|w\|_{2}$.

2. The map $u, w \rightarrow v(u, w)$ is locally Lipschitz from $H_{0}^{2}(\Omega) \times H_{0}^{2}(\Omega) \rightarrow W^{2, \infty}(\Omega)$.

This lemma easily implies that $f(u)$ in (2.14) given by $f(u)=-\left[u, v(u)+F_{0}\right]$ is locally Lipschitz.

Since the topology for the velocity $u_{t}$ is now $L_{2}(\Omega)$, (rather than $H_{0}^{1}(\Omega)$, as in the rotational case $\gamma>0$ ), we use inequality (2.15) rather than (3.3.5) p. 189 [14. This modification allows us to repeat the arguments in [14, in order to conclude with the statements of Proposition 2.10 For this we also make use of the first part of the following lemma:

Lemma 2.12. We denote $q^{u}(t)=q\left(u^{t}, t\right)$. Let Assumption 2.7 be in force. Then

$$
\left|\int_{0}^{t}<q^{u}(\tau), u_{t}(\tau)>d \tau\right| \leq C \epsilon^{-1} t^{*} \int_{-t^{*}}^{t}\|u(\tau)\|_{2}^{2} d \tau+\epsilon \int_{0}^{t}\left\|u_{t}(\tau)\right\|^{2} d \tau, \quad \forall \epsilon>0, \forall t \in[0, T],
$$

for any $u \in L_{2}\left(-t^{*}, T ; H^{2}(\Omega)\right) \cap W_{2}^{1}\left(0, T ; L_{2}(\Omega)\right)$.

If, in addition, we assume Assumption [2.8, then there exists $\eta_{*}>0$ such that for every $\epsilon>0$ we have the estimate:

$$
\left|\int_{0}^{t}<q^{u}(\tau), u_{t}(\tau)>d \tau\right| \leq \epsilon \int_{-t^{*}}^{t}\|u(\tau)\|_{2}^{2} d \tau+C\left(t^{*}, \epsilon\right) \cdot(1+T) \sup _{[0, t]}\|u(\tau)\|_{2-\eta_{*}}^{2}, \quad \forall t \in[0, T],
$$

for any $u \in L_{2}\left(-t^{*}, T ; H^{2}(\Omega)\right) \cap C\left(0, T ; H^{2-\eta_{*}}(\Omega)\right) \cap C^{1}\left(0, T ; L_{2}(\Omega)\right)$.

For the proof we refer to Section 6 .

In order to consider the delayed system as a dynamical system with the phase space $\mathbf{H}$ we recall the notation: $u^{t}(s) \equiv u(t+s), s \in\left[-t^{*}, 0\right]$. With the above notation we introduce the operator $S_{t}: \mathbf{H} \mapsto \mathbf{H}$ by the formula

$$
S_{t}\left(u_{0}, u_{1}, \eta\right) \equiv\left(u(t), u_{t}(t), u^{t}\right),
$$

where $u(t)$ solves (2.14). Proposition 2.10 implies the following conclusion 
Corollary 2.13. $S_{t}: \mathbf{H} \rightarrow \mathbf{H}$ is a strongly continuous semigroup on $\mathbf{H}$.

Proof. Strong continuity is stated in Proposition 2.10. The semigroup property follows from uniqueness. To prove continuity with respect to initial data we use the following assertion.

Lemma 2.14. Suppose $u^{i}(t)$ for $i=1,2$ are weak solutions to 2.14 with different initial data and $z=u^{1}-u^{2}$. Additionally assume that

$$
\left\|u_{t}^{i}(t)\right\|^{2}+\left\|\Delta u^{i}(t)\right\|^{2} \leq R^{2}, i=1,2
$$

for some $R>0$ and all $t \in[0, T]$. Then there exists $C>0$ and $a_{R} \equiv a_{R}\left(t^{*}\right)>0$ such that

$$
\left\|z_{t}(t)\right\|^{2}+\|\Delta z(t)\|^{2} \leq C e^{a_{R} t}\left\{\left\|\Delta\left(u_{0}^{1}-u_{0}^{2}\right)\right\|^{2}+\left\|u_{1}^{1}-u_{1}^{2}\right\|^{2}+\int_{-t^{*}}^{0}\left\|\eta^{1}(\tau)-\eta^{2}(\tau)\right\|_{2}^{2} d \tau\right\}
$$

for all $t \in[0, T]$.

Proof. We have that $z$ solves the following problem

$$
\left\{\begin{array}{l}
z_{t t}+\Delta^{2} z+k z_{t}+f\left(u^{1}\right)-f\left(u^{2}\right)=q^{z}(t)-L z \\
z=\partial_{\nu} z=0 \text { on } \partial \Omega \\
z(0)=z_{0} \in H_{0}^{2}(\Omega), z_{t}(0)=z_{1} \in L_{2}(\Omega),\left.z\right|_{\left(-t^{*}, 0\right)} \in L_{2}\left(-t^{*}, 0 ; H_{0}^{2}(\Omega)\right)
\end{array}\right.
$$

where as above we denote $q^{z}(t)=q\left(z^{t}, t\right)$. Let

$$
E_{z}(t) \equiv \frac{1}{2}\left\{\|\Delta z(t)\|^{2}+\left\|z_{t}(t)\right\|^{2}\right\}
$$

then making use of the the energy equality for the difference $z$

$$
\begin{aligned}
E_{z}(t)+k \int_{0}^{t}\left\|z_{t}\right\|^{2} d \tau= & E_{z}(0)-\int_{0}^{t}<f\left(u^{1}\right)-f\left(u^{2}\right), z_{t}>d \tau+\int_{0}^{t}<q^{z}(\tau), z_{t}(\tau)>d \tau \\
& -\int_{0}^{t}<L z(\tau), z_{t}(\tau)>d \tau
\end{aligned}
$$

we have the following:

$$
E_{z}(t)+k \int_{0}^{t}\left\|u_{t}\right\|^{2} d \tau \leq E_{z}(0)+\int_{0}^{t}\left[\left\|f\left(u^{1}\right)-f\left(u^{2}\right)\right\|+\|L z\|\right]\left\|z_{t}\right\| d \tau|+| \int_{0}^{t}<q^{z}, z_{t}>d \tau \mid .
$$

From here, we apply the estimates in (2.21) for $q^{z}$ with $\epsilon=1 / t^{*}$, and note the locally Lipschitz character of the von Karman nonlinearity $f(u)$, yielding

$$
E_{z}(t)+k \int_{0}^{t}\left\|u_{t}\right\|^{2} d \tau \leq C_{0}\left(E_{z}(0)+\int_{-t^{*}}^{0}\|z(\tau)\|_{2}^{2} d \tau\right)+C\left(R, t^{*}\right) \int_{0}^{t} E_{z}(\tau) d \tau .
$$

Hence, Gronwall's inequality yields the desired estimate in (2.25). 
Using Lemma 2.14 we obtain that

$$
\left\|S_{t} y_{1}-S_{t} y_{2}\right\|_{\mathbf{H}}^{2} \leq C e^{a_{R} t}\left\|y_{1}-y_{2}\right\|_{\mathbf{H}}^{2}
$$

for $S_{t} y_{i}=\left(u^{i}(t), u_{t}^{i}(t),\left[u^{i}\right]^{t}\right)$ with $y_{i}=\left(u_{0}^{i}, u_{1}^{i}, \eta\right)$, such that (2.24) holds. This allows us to conclude the proof of Corollary 2.13.

\section{Statement of Main Results}

Our main results deal with (1) long-time dynamics of the system $\left(S_{t}, \mathbf{H}\right)$ generated by (2.14) and (2) its connection with the flow-structure dynamics governed by (2.6).

Theorem 3.1. Let both Assumptions 2.7 and 2.8 be in force and $\left(S_{t}, \mathbf{H}\right)$ be the dynamical system generated weak solutions to the system in (2.14) with $k>0$ on the space $\mathbf{H} \equiv H_{0}^{2}(\Omega) \times L_{2}(\Omega) \times$ $L_{2}\left(-t^{*}, 0 ; H_{0}^{2}(\Omega)\right)$ with evolution operator given by (2.23). Then the system $\left(S_{t}, \mathbf{H}\right)$ has a compact global attractor $\mathbf{A}$ of finite fractal dimension. The attractor can be characterized as the set of all bounded full trajectories. Moreover, the set $\mathbf{A}$ has additional regularity; namely, any full trajectory $y(t)=\left(u(t), u_{t}(t), u^{t}\right) \subset \mathbf{A}, t \in \mathbb{R}$, has the property that $u \in L_{\infty}\left(\mathbb{R} ; H^{4}(\Omega) \cap H_{0}^{2}(\Omega)\right)$ and $u_{t} \in$ $L_{\infty}\left(\mathbb{R} ; H^{2}(\Omega)\right)$.

We recall (see, e.g., 1, 9, 28, 34]) that a global attractor $\mathbf{A}$ is a closed, bounded set in $\mathbf{H}$ which is invariant (i.e., $S_{t} \mathbf{A}=\mathbf{A}$ for all $t>0$ ) and uniformly attracts every bounded set $B$, i.e.

$$
\lim _{t \rightarrow+\infty} d_{\mathbf{H}}\left\{S_{t} B \mid \mathbf{A}\right\}=0, \quad \text { where } d_{\mathbf{H}}\left\{S_{t} B \mid \mathbf{A}\right\} \equiv \sup _{y \in B} \operatorname{dist}_{\mathbf{H}}(y, \mathbf{A}),
$$

for any bounded $B \in \mathbf{H}$.

The fractal (box-counting) dimension $\operatorname{dim}_{f} \mathbf{A}$ of $\mathbf{A}$ is defined by

$$
\operatorname{dim}_{f} \mathbf{A}=\limsup _{\epsilon \rightarrow 0} \frac{\ln n(\mathbf{A}, \epsilon)}{\ln (1 / \epsilon)},
$$

where $n(M, \epsilon)$ is the minimal number of closed balls in $\mathbf{H}$ of the radius $\epsilon$ which cover the set $M$.

Remark 3.2. We note that this type of additional regularity of solutions from the attractor mentioned in Theorem 3.1 is not possible in the case $\gamma>0$, owing to the fact that the principal term in the equation is $\Delta^{2} u-\gamma \Delta u_{t t}$. In this case, the presence of this term disallows the use of elliptic regularity theory (applied to the biharmonic term) on elements of the attractor. More importantly, when $\gamma>0$, in order to obtain the strong attractiveness property a much stronger damping is necessary. In order to stabilize the kinetic part of the energy one will have to introduce $-\gamma \Delta u_{t}$ as a damping term (see section 9.3 in [14]). The point we want to stress is that in our case only $u_{t}$ as a damping term is needed. And, in fact, it is this term that will be generated by the flow from "thin air". As a consequence, the plate (in the full flow-plate system) will require no mechanical damping at all.

Having established the quasicompact character of the delayed potential $q^{u}$ as in (2.8) (showing that it satisfies the conditions (2.15)-(2.17) we can now apply Theorem 3.1 to the von Karman flow-plate model in (2.6). 
Theorem 3.3. Suppose $0 \leq U \neq 1, k \geq 0, F_{0} \in H^{3+\delta}(\Omega)$ and $p_{0} \in L_{2}(\Omega)$. Then there exists a compact set $\mathscr{U} \subset H_{0}^{2}(\Omega) \times L_{2}(\Omega)=\mathcal{H}$ of finite fractal dimension such that

$$
\lim _{t \rightarrow \infty} d_{\mathcal{H}}\left(\left(u(t), u_{t}(t)\right), \mathscr{U}\right)=\lim _{t \rightarrow \infty} \inf _{\left(\nu_{0}, \nu_{1}\right) \in \mathscr{U}}\left(\left\|u(t)-\nu_{0}\right\|_{2}^{2}+\left\|u_{t}(t)-\nu_{1}\right\|^{2}\right)=0
$$

for any weak solution $\left(u, u_{t} ; \phi, \phi_{t}\right)$ to (2.6) with initial data

$$
\left(u_{0}, u_{1} ; \phi_{0}, \phi_{1}\right) \in H \equiv H_{0}^{2}(\Omega) \times L_{2}(\Omega) \times H^{1}\left(\mathbb{R}_{+}^{3}\right) \times L_{2}\left(\mathbb{R}_{+}^{3}\right)
$$

which are localized in $\mathbb{R}_{+}^{3}$ (i.e., $\phi_{0}(\mathbf{x})=\phi_{1}(\mathbf{x})=0$ for $|\mathbf{x}|>R$ for some $R>0$ ). Additionally, we have the additional regularity $\mathscr{U} \subset\left(H^{4}(\Omega) \cap H_{0}^{2}(\Omega)\right) \times H^{2}(\Omega)$.

Proof. The proof of this result follows from rewriting the dynamical system $\left(T_{t}, H\right)$ generated by (2.6) as the delayed system in (2.7). The latter is possible for sufficiently large times by Theorem 2.3 . Since the delayed potential $q^{u}$ was shown to satisfy Assumptions 2.7 and 2.8 we may apply our main result, Theorem 3.1 to the dynamical system generated by the weak solution to (2.7) on the space $\mathbf{H}=H_{0}^{2}(\Omega) \times L_{2}(\Omega) \times L_{2}\left(-t^{*}, 0 ; H_{0}^{2}(\Omega)\right)$. This yields a compact global attractor $\mathbf{A} \subset \mathbf{H}$ of finite dimension and additional regularity; we then take $\mathscr{U}$ to be the projection of $\mathbf{A}$ on $H$, which concludes the proof as in [14.

Remark 3.4. We here reiterate that the above result holds in the absence of imposed damping, i.e., with $k=0$ in (2.6). Utilizing the natural damping appearing in the reduced flow pressure, we see that in the case of $\gamma=0$, the flow naturally provides a stabilizing effect to the dynamics in that it yields the existence of the compact attractor. This is not the case when $\gamma>0$, as the nature of the damping must be (necessarily) stronger (see Remark 3.2).

Remark 3.5. It should also be noted here that because we have rewritten our problem (2.6) as a reduced delayed plate, and additionally changed the state space upon which we are operating, the results obtained on long-time behavior will not be invariant with respect to the flow component of the model, i.e. our global attractors will be with respect to the state space $\mathbf{H}$, as defined above. Again, the data in the form of the delayed term $\left.u\right|_{\left(-t^{*}, 0\right)}$ contains the information from the flow itself. Obtaining global attractors for the full state space corresponding to $\left(u, u_{t} ; \phi, \phi_{t}\right) \in H_{0}^{2}(\Omega) \times L_{2}(\Omega) \times$ $H^{1}\left(\mathbb{R}_{+}^{3}\right) \times L_{2}\left(\mathbb{R}_{+}^{3}\right)$ is not a realistic task from the mathematical point of view. There is no damping imposed on the system, thus the flow component evolves according to the full half space, unconstrained dynamics. The obtained result on the structure (without damping) is the best possible result with respect to both the underlying physics and mathematics of the problem.

Previous Literature and New Challenges: Nonlinear PDEs with delays have been considered in various sources (see [35] and references therein). In relation to plate equations with delayed aerodynamical type pressure, we note that 14 provides a rather complete analysis of the delayed von Karman plate in the presence of rotational terms (and application to flow-plate interactions), and the aforementioned references [7, 17] deal with the plate with delay in the presence of the Berger nonlinearity. In this latter references, it is assumed that the parameter in front of delayed term is suitably small. The analysis in the more recent reference [14] also, by and large, applies to the Berger plate (in the rotational case). 
One should stress at the outset that the problem is challenging, even in the rotational case. This is due to the fact that the underlying system is intrinsically non-gradient (both the delay term and the term $U \partial_{x} u$ provide non-conservative and non-dissipative terms that contribute to the loss of gradient structure). In view of this, the existence of attractors requires a priori information on a uniformly absorbing set. The presence of delay terms along with non-conservative terms makes the latter task challenging [7].

The references pertaining to plates with delayed terms primarily with the rotational case, i.e. the plate equation discussed in Remark 2.6. In particular, for $\gamma, k_{1}, k_{2}>0$, well-posedness of weak solutions is established via the Galerkin method [14, Sections 3.3.1, 4.1.6]. These solution are shown to generate a dynamical system the state space $\mathbf{H} \equiv H_{0}^{2}(\Omega) \times H_{0}^{1}(\Omega) \times L_{2}\left(-t^{*}, 0 ; H_{0}^{2}(\Omega)\right)$. Then, exploiting the compactness of the term $\left\langle q^{u}, u_{t}\right\rangle$, i.e. making use of the duality pairing $\left(H_{0}^{1}, H^{-1}\right)$, dissipativity of the dynamical system can be shown (in much the same way which we utilize below), followed by asymptotic smoothness. In this case, however, asymptotic smoothness is arrived at in a straightforward way, which additionally exploits the compactness of the von Karman nonlinearity (with respect to the energy identity) in the case where $u_{t}$ in $H_{0}^{1}(\Omega)$.

The mathematical hurdles arising in the $\gamma=0$ case (where $u_{t} \in L_{2}(\Omega)$ ) begin at the outset with well-posedness of weak solutions; indeed, many well-posedness and long-time behavior analysis [36, 30, 16] are dramatically complicated when $\gamma=0$. Thus, it is no wonder that, to date, the nonrotational von Karman plate with delayed terms has not been considered. The reasons for this are clear: (i) the methods of studying long-time behavior via an approach making use of the combination of Theorems 4.1 and 4.2 (stated below) is relatively recent. In particular, dealing with the von Karman nonlinearity is especially difficult outside of the use of Theorem 4.2 (ii) In addition, the initial studies [8] of delayed von Karman plates took place before results on the sharp regularity of the Airy stress function were available. These results are critical in this treatment. (iii) Lastly, it is clear by inspection that additional properties of the delayed force in (2.14) must be accounted for when the rotational inertia term is absent. However, owing to a gap in well-posedness results for flow-plate interactions, it was unclear what these properties - translated into abstract assumptions - should be. A recent observation about the 'hidden compactness' of the reduced delayed potential (derived from an inviscid potential flow) yielded insight into what assumptions are reasonable in line with previous analysis of von Karman plates with delay.

In proving finite-dimensionality and smoothness of the attractors, the criticality of the nonlinearity and the lack of gradient structure prevents one from using a powerful technique of backward smoothness of trajectories [1, 26, 14, where smoothness is propagated forward from the equilibria. Since the attractor may have complicated structure, the structure of the attractor is not characterized by the equilibria points. In order to cope with this issue, we take the advantage of novel method that is based on density and exploits only the compactness of the attractor.

\section{Proof of Main Result}

We first outline the steps utilized to obtain the main result stated above.

- We begin with recalling the key results on dissipative long-time dynamics for non-gradient systems. 
- We then recall (in the form adapted to the delay case) cite the primary estimates which have been used in previous long-time behavior considerations for von Karman plates in the past. Noting that we cannot make use of the $\gamma>0$ approach to long-time behavior (owing to the loss of compactness of the term $<q^{u}(t), u_{t}(t)>$ ). We begin by exploiting a different assumption ('hidden' compactness of this term when integrated in $t$ in the energy relation (2.18)).

- We then use a similar, modified Lyapunov functional as that in [14, p.480] on the dynamical system to show that it is dissipative.

- After obtaining the necessary compactness estimates, we synthesize them to produce a pointwise energy estimate which allows us to make use the abstract Theorem 4.2 to obtain asymptotic smoothness of the dynamical system associated with weak solutions to (2.14). At this point, we make use of abstract Theorem 4.1 to conclude that the dynamical system possesses a compact attractor in the space $\mathbf{H}$.

- In the last step, we revisit our estimation in the asymptotic smoothness section to obtain the socalled quasistability estimate on the attractor utilizing its compactness; this allows us to apply Theorem 4.3. The application of this theorem gives the finite dimensionality and additional smoothness of the attractor.

\subsection{Preliminaries on Dissipative Dynamical Systems}

We recall notions and results from the theory of dynamical systems (see, e.g., [1, 9, 28, 34]).

One says that a dynamical system $\left(S_{t}, \mathbf{H}\right)$ is asymptotically smooth if for any bounded, forward invariant set $D$ there exists a compact set $K \subset \bar{D}$ such that

$$
\lim _{t \rightarrow+\infty} d_{\mathbf{H}}\left\{S_{t} D \mid K\right\}=0
$$

holds. An asymptotically smooth dynamical system should be thought of as one which possesses local attractors, i.e. for a given forward invariant set $B_{R}$ of diameter $R$ in the space $\mathbf{H}$ there exists a compact attracting set in the closure of $B_{R}$, however, this set need not be uniform with respect to $R$.

A closed set $B \subset \mathbf{H}$ is said to be absorbing for $\left(S_{t}, \mathbf{H}\right)$ if for any bounded set $D \subset \mathbf{H}$ there exists a $t_{0}(D)$ such that $S_{t} D \subset B$ for all $t>t_{0}$. If the dynamical system $\left(S_{t}, \mathbf{H}\right)$ has a bounded absorbing set it is said to be dissipative.

In the context of this paper we will use a few keys theorems (which we now formally state) to prove the existence of a finite dimensional global attractor. First, we address the existence of attractors and characterize the attracting set:

Theorem 4.1. Any dissipative and asymptotically smooth dynamical system $\left(S_{t}, \mathbf{H}\right)$ in a Banach space $\mathbf{H}$ possesses a unique compact global attractor $\boldsymbol{A}$. This attractor is a connected set and can be described as a set of all bounded full trajectories.

For the proof, see [1] or 34].

Secondly, we state a useful criterion (inspired by [26] and proven in [12, see also [14, Chapter 7]) which reduces asymptotic smoothness to finding a suitable functional on the state space with a compensated compactness condition: 
Theorem 4.2. Let $\left(S_{t}, \mathbf{H}\right)$ be a dynamical system. Assume that for any bounded positively invariant set $B \subset \mathbf{H}$ and for all $\epsilon>0$ there exists a $T \equiv T_{\epsilon, B}$ such that

$$
\left\|S_{T} x_{1}-S_{T} x_{2}\right\|_{\mathbf{H}} \leq \epsilon+\Psi_{\epsilon, B, T}\left(x_{1}, x_{2}\right), \quad x_{i} \in B
$$

with $\Psi$ a functional defined on $B \times B$ depending on $\epsilon, T$, and $B$ such that

$$
\liminf _{m} \liminf _{n} \Psi_{\epsilon, T, B}\left(x_{m}, x_{n}\right)=0
$$

for every sequence $\left\{x_{n}\right\} \subset B$. Then $\left(S_{t}, \mathbf{H}\right)$ is an asymptotically smooth dynamical system.

In order to establish both smoothness of the attractor and finite dimensionality, a stronger estimate on the difference of two flows is needed. We now cite [14, pp. 381-387] and also [15]. Note that we have used a specialization of the cited theorem which utilizes the special structure of the state space in the problem at hand: $\left.\mathbf{H}=H_{0}^{2}(\Omega) \times L_{2}(\Omega) \times L_{2}\left(-t^{*}, 0 ; H_{0}^{2}(\Omega)\right)\right)$; the theorems cited above are more general:

Theorem 4.3. Let $\mathbf{A}$ be a global attractor for $\left(S_{t}, \mathbf{H}\right)$.

$x_{1}, x_{2} \in B \subset \mathbf{H}$ where $B$ is a forward invariant set for the flows $S_{t} x_{i}$. Assume that the following inequality holds for all $t>0$ with positive constants $C_{1}, C_{2}, \omega$

$$
\left\|S_{t} x_{1}-S_{t} x_{2}\right\|_{\mathbf{H}}^{2} \leq C_{1} e^{-\omega_{B} t}\left\|x_{1}-x_{2}\right\|_{\mathbf{H}}^{2}+C_{2} \max _{\tau \in[0, t]}\left\|u_{1}(\tau)-u_{2}(\tau)\right\|_{H_{1}}^{2}
$$

for any $x_{1}, x_{2} \in \mathbf{A}$, where $S_{t} x_{i}=\left(u_{i}(t), \partial_{t} u_{i}(t), u_{i}^{t}\right)$ and $H_{0}^{2}(\Omega) \subset H_{1} \subset L_{2}(\Omega)$ is compactly embedded. Then the attractor $\mathbf{A}$ posesses the following properties:

(a) The fractal dimension of $\mathbf{A}$ is finite.

(b) For any $x \in \mathbf{A}$ one has $\partial_{t}\left(S_{t} x\right) \in L_{\infty}(\mathbb{R}, \mathbf{H})$.

The estimate in (4.1) is often referred to (in practice) as a "quasistability" estimate. It reflects the fact that the flow can be stabilized exponentially to a compact set. Alternatively, we might say that the flow is exponentially stable, modulo a compact perturbation (lower order terms). The quadratic nature of the lower order terms is important for the validity of Theorem 4.3 .

\subsection{Technical Preliminaries}

In this section we derive and cite certain energy and multiplier estimates, as well as estimates on the von Karman nonlinearity, which will be necessary in the proof of Theorem 3.1 below.

The following theorem is a case specialization found in [14, Section 1.4, pp.38-45; Section 9.4, pp.496-497]. These bounds elucidate the local Lipschitz (quasi-Lipschitz) character of the von Karman nonlinearity are relatively recent and critical to our nonlinear analysis.

Theorem 4.4. Let $u^{i} \in \mathscr{B}_{R}\left(H_{0}^{2}(\Omega)\right), i=1,2$, and $z=u^{1}-u^{2}$. Then for $f(u)=-\left[u, v(u)+F_{0}\right]$ we have

$$
\left\|f\left(u^{1}\right)-f\left(u^{2}\right)\right\|_{-\delta} \leq C_{\delta}\left(1+\left\|u^{1}\right\|_{2}^{2}+\left\|u^{2}\right\|_{2}^{2}\right)\|z\|_{2-\delta} \leq C(\delta, R)\|z\|_{2-\delta} \quad \text { for all } \delta \in[0,1] .
$$


If we further assume that $u^{i} \in C\left(s, t ; H^{2}(\Omega)\right) \cap C^{1}\left(s, t ; L_{2}(\Omega)\right)$, then we have that

$$
-<f\left(u^{1}\right)-f\left(u^{2}\right), z_{t}>=\frac{1}{4} \frac{d}{d t} Q(z)+\frac{1}{2} P(z)
$$

where

$$
Q(z)=<v\left(u^{1}\right)+v\left(u^{2}\right),[z, z]>-\left\|\Delta v\left(u^{1}+u^{2}, z\right)\right\|^{2}
$$

and

$$
P(z)=-<u_{t}^{1},\left[u^{1}, v(z)\right]>-<u_{t}^{2},\left[u^{2}, v(z)\right]>-<u_{t}^{1}+u_{t}^{2},\left[z, v\left(u^{1}+u^{2}, z\right)\right]>.
$$

Moreover,

$$
\left|\int_{s}^{t}<f\left(u^{1}(\tau)\right)-f\left(u^{2}(\tau)\right), z_{t}(\tau)>d \tau\right| \leq C(R) \sup _{\tau \in[s, t]}|| z \|_{2-\eta}^{2}+\frac{1}{2}\left|\int_{s}^{t} P(z) d \tau\right|
$$

for some $0<\eta<1 / 2$ provided $u^{i}(\tau) \in \mathscr{B}_{R}\left(H_{0}^{2}(\Omega)\right)$ for all $\tau \in[s, t]$.

The above bounds rely on the equation

$$
f\left(u^{1}\right)-f\left(u^{2}\right)=\left[z, v(u)+F_{0}\right]+\left[u^{2}, v\left(u^{1}\right)-v\left(u^{2}\right)\right]
$$

and on the so-called 'sharp' regularity of the Airy stress function $v(u)$ (see Lemma 2.11).

We will now make use of the above estimates in producing energy type estimates.

First, we multiply (2.14) by $u$ and integrate over the set $\Omega \times(s, t)$, making use of clamped boundary conditions. This produces the following identity:

$$
\begin{aligned}
<u_{t}(\tau), u(\tau)>\left.\right|_{s} ^{t}+ & \int_{s}^{t}\left(\|\Delta u(\tau)\|^{2}-\left\|u_{t}(\tau)\right\|^{2}\right) d \tau \\
& =-\int_{s}^{t}\left(<f(u(\tau)), u(\tau)>+<q^{u}(\tau)+p_{0}-L u, u(\tau)>\right) d \tau
\end{aligned}
$$

where as above we use the notation $q^{u}(\tau)=q\left(u^{\tau}, \tau\right)$. By standard splitting and interpolation, we arrive at

$$
\begin{aligned}
\int_{s}^{t}\left(\|\Delta u(\tau)\|^{2}-\left\|u_{t}(\tau)\right\|^{2}\right) d \tau \leq & \epsilon \int_{s}^{t}\|u\|_{2}^{2} d \tau+\epsilon\|p+0\|^{2}-\int_{s}^{t}<f(u(\tau)), u(\tau)>d \tau \\
& +C \int_{s}^{t}\left\|q^{u}(\tau)\right\|_{-\sigma}^{2} d \tau+C_{\epsilon}(1+|t-s|) \sup _{\tau \in[s, t]}\|u(\tau)\|_{2-\eta}^{2} \\
& +\left|<u_{t}(t), u(t)>\right|+\left|<u_{t}(s), u(s)>\right|
\end{aligned}
$$

for all $\epsilon>0$ and for some $\eta>0$. This estimate, coupled with the estimates in (2.16) and (4.2) yield the following estimates:

Lemma 4.5. Let $u^{i} \in C\left(0, T ; H_{0}^{2}(\Omega)\right) \cap C^{1}\left(0, T ; L_{2}(\Omega)\right) \cap L_{2}\left(-t^{*}, T ; H_{0}^{2}(\Omega)\right)$ solve 2.14 with clamped boundary conditions and appropriate initial conditions on $[0, T]$ for $i=1,2$. Then the following 
estimate holds for all $\epsilon>0$, for some $\eta>0$, and $0 \leq t \leq T$ :

$$
\begin{aligned}
\int_{0}^{t}\left(\|\Delta u\|^{2}-\left\|u_{t}\right\|^{2}\right) d \tau \leq & \epsilon \int_{0}^{t}\|u\|_{2}^{2} d \tau+\epsilon+C \int_{-t^{*}}^{0}\|u(\tau)\|_{2}^{2} d \tau+C\left(\epsilon, t^{*}, T\right) \sup _{\tau \in[0, t]}\|u(\tau)\|_{2-\eta}^{2} \\
& -\int_{0}^{t}<f(u), u>d \tau+\left|<u_{t}(t), u(t)>\right|+\left|<u_{t}(s), u(s)>\right| .
\end{aligned}
$$

Moreover, in the case where we are considering the difference $z=u^{1}-u^{2}$ of solutions solving (2.26) with $u^{i}(t) \in \mathscr{B}_{R}\left(H^{2}(\Omega)\right)$ for all $t \in[0, T]$, we may utilize the estimates in Theorem 4.4 (which eliminates the stand-alone $\epsilon$ ) arrive at

$$
\begin{aligned}
\int_{s}^{t}\left(\|\Delta z\|^{2}-\left\|z_{t}\right\|^{2}\right) d \tau \leq & \epsilon \int_{s}^{t}\|z\|_{2}^{2} d \tau+C \int_{s-t^{*}}^{t}\|z(\tau)\|_{2-\sigma}^{2} d \tau+C(\epsilon, T, R) \sup _{\tau \in[0, t]}\|z(\tau)\|_{2-\eta}^{2} \\
& +E_{z}(t)+E_{z}(s)
\end{aligned}
$$

where $E_{z}(t)$ is given by (2.27), i.e., $E_{z}(t) \equiv \frac{1}{2}\left\{\|\Delta z(t)\|^{2}+\left\|z_{t}(t)\right\|^{2}\right\}$.

The final class of estimates we need are energy estimates for the $z$ term defines as the solution to (2.26). Energy estimates for single solutions (making use of the nonlinear potential energy) can be derived straightforwardly from (2.18). The energy estimate on $z$, along with the estimate in (4.5) above, will be used in showing asymptotic smoothness for the system.

The energy relation on $[s, t]$ for $z$ in (2.26) is given by

$$
\begin{aligned}
E_{z}(t)+k \int_{s}^{t}\left\|z_{t}\right\|^{2} d \tau= & E_{z}(s)-\int_{s}^{t}<f\left(u^{1}\right)-f\left(u^{2}\right), z_{t}>d \tau+\int_{s}^{t}<q^{z}(\tau), z_{t}(\tau)>d \tau \\
& -\int_{s}^{t}<L z(\tau), z_{t}(\tau)>d \tau
\end{aligned}
$$

From this, making use of splitting and Sobolev inequalities, we quickly have for $0 \leq s<t \leq T$, some $\eta>0$, and all $\epsilon>0$ :

$$
\begin{aligned}
E_{z}(t)+k \int_{s}^{t}\left\|z_{t}\right\|^{2} d \tau \leq & E_{z}(s)+C(\epsilon, T) \sup _{\tau \in[s, t]}\|z\|_{2-\eta}^{2}+\epsilon \int_{s}^{t}\left(\|z\|_{2}^{2}+\left\|z_{t}\right\|^{2}\right) d \tau \\
& -\int_{s}^{t}<f\left(u^{1}\right)-f\left(u^{2}\right), z_{t}>d \tau+\left|\int_{s}^{t}<q^{z}(\tau), z_{t}(\tau)>d \tau\right|
\end{aligned}
$$

In the case when $k>0$ by (4.5) this implies that

$$
\begin{aligned}
\frac{1}{2} E_{z}(t)+c_{0} \int_{s}^{t} E_{z} d \tau \leq & E_{z}(s)+C(T, R) \sup _{\tau \in[s, t]}\|z\|_{2-\eta}^{2}+C \int_{s-t^{*}}^{t}\|z(\tau)\|_{2-\sigma}^{2} d \tau \\
& -\int_{s}^{t}<f\left(u^{1}\right)-f\left(u^{2}\right), z_{t}>d \tau+\left|\int_{s}^{t}<q_{t}^{z}(\tau), z(\tau)>d \tau\right| \\
& +\left|<q^{z}(t), z(t)>\right|+\left|<q^{z}(s), z(s)>\right| .
\end{aligned}
$$

Here above we usee the integration by parts formula for the integral with delayed term. Therefore 
there exist $a_{i}>0$ and $C(T, R)>0$ such that

$$
\begin{aligned}
E_{z}(t)+\int_{s}^{t} E_{z} d \tau \leq & a_{0}\left(E_{z}(s)+\int_{s-t^{*}}^{s}\|z(\tau)\|_{2-\sigma}^{2} d \tau\right)+C(T, R) \sup _{\tau \in[s, t]}\|z\|_{2-\eta_{*}}^{2} \\
& -a_{1} \int_{s}^{t}<f\left(u^{1}\right)-f\left(u^{2}\right), z_{t}>d \tau .
\end{aligned}
$$

Taking $t=T$ and integrating over $s$ in $[0, T]$ we arrive at 3

$$
\begin{aligned}
T E_{z}(T)+ & \int_{0}^{T} d s \int_{s}^{T} E_{z} d \tau \leq a_{0}\left(E_{z}(0)+\int_{-t^{*}}^{0}\|z(\tau)\|_{2-\sigma}^{2} d \tau\right)+C(T, R) \sup _{\tau \in[0, T]}\|z\|_{2-\eta_{*}}^{2} \\
& -a_{1} \int_{0}^{T} d s \int_{s}^{T}<f\left(u^{1}\right)-f\left(u^{2}\right), z_{t}>d \tau-a_{2} \int_{0}^{T}<f\left(u^{1}\right)-f\left(u^{2}\right), z_{t}>d \tau .
\end{aligned}
$$

Since

$$
\int_{0}^{T} d s \int_{s}^{T} E_{z} d \tau \geq \frac{T}{2} \int_{T-t^{*}}^{T} E_{z} d \tau \quad \text { for } T \geq 2 t^{*}
$$

we arrive to the following assertion.

Lemma 4.6. Let $u^{i} \in C\left(0, T ; H_{0}^{2}(\Omega)\right) \cap C^{1}\left(0, T ; L_{2}(\Omega)\right) \cap L_{2}\left(-t^{*}, T ; H_{0}^{2}(\Omega)\right)$ solve 2.14) with clamped boundary conditions and appropriate initial conditions on $[0, T]$ for $i=1,2, T \geq 2 t^{*}$. Additionally, assume $u^{i}(t) \in \mathscr{B}_{R}\left(H^{2}(\Omega)\right)$ for all $t \in[0, T]$. Then the following estimates

$$
\begin{aligned}
\frac{T}{2}\left[E_{z}(T)+\right. & \left.\int_{T-t^{*}}^{T} E_{z}(\tau) d \tau\right] \leq a_{0}\left(E_{z}(0)+\int_{-t^{*}}^{0}\|z(\tau)\|_{2}^{2} d \tau\right)+C(T, R) \sup _{\tau \in[0, T]}\|z\|_{2-\eta_{*}}^{2} \\
& -a_{1} \int_{0}^{T} d s \int_{s}^{T}<f\left(u^{1}\right)-f\left(u^{2}\right), z_{t}>d \tau-a_{2} \int_{0}^{T}<f\left(u^{1}\right)-f\left(u^{2}\right), z_{t}>d \tau
\end{aligned}
$$

hold with $a_{i}$ independent of $T$ and $R$.

\subsection{Dissipative Dynamical System}

Our next task in order to to make use of Theorem 4.1 is to show dissipativity of the dynamical system $\left(S_{t}, \mathbf{H}\right)$, namely that there exists a bounded, forward invariant, absorbing set. To show this, similar to the consideration in [14, Theorem 9.3.4, p.480], we consider the Lyapunov type function (with $\mathscr{E}\left(u, u_{t}\right)$ as in (2.19) and with $\Pi_{*}(u)$ given by (2.20)

$$
\begin{aligned}
V\left(S_{t} y\right) \equiv & \mathscr{E}\left(u(t), u_{t}(t)\right)-<q^{u}(t), u(t)>+\nu\left(<u_{t}, u>+\frac{k}{2}\|u\|^{2}\right) \\
& +\mu\left(\int_{t-t^{*}}^{t} \Pi_{*}(u(s)) d s+\int_{0}^{t^{*}} d s \int_{t-s}^{t} \Pi_{*}(u(\tau)) d \tau\right)
\end{aligned}
$$

\footnotetext{
${ }^{3}$ With other constants $a_{i}$ and $C(T, R)$.
} 
where $S_{t} y \equiv y(t)=\left(u(t), u_{t}(t), u^{t}\right)$ for $t \geq 0$ and $\mu, \nu$ are some positive numbers to be specified below. In view of the results for the von Karman plate in [14, Section 4.1.1], we have that

$$
c_{0} E\left(u, u_{t}\right)-c \leq V\left(S_{t} y\right) \leq c_{1} E\left(u, u_{t}\right)+\mu C t^{*} \int_{-t^{*}}^{0} \Pi_{*}(u(t+\tau)) d \tau+c
$$

for $\nu>0$ small enough, where $c_{0}, c_{1}, c, C>0$ are constant. Here we make use of the notation: $E\left(u, u_{t}\right) \equiv \frac{1}{2}\left\|u_{t}\right\|^{2}+\Pi_{*}(u)$.

To obtain the above bound, we make direct use of our assumption on the $L_{2}$ bound in (2.16) on the term $\left\langle q^{u}, u\right\rangle$. Additionally, we here need (and below) a critical lower bound on the potential energy, which can be found in various forms throughout [14, p. 49 and p. 132]:

Proposition 4.7. For any $u \in H^{2}(\Omega) \cap H_{0}^{1}(\Omega)$ we have for any $\delta>0$ and any $0<\eta \leq 2$

$$
\|u\|_{2-\eta}^{2} \leq \delta\left(\|u\|_{2}^{2}+\|\Delta v(u)\|^{2}\right)+C_{\delta},
$$

where $v(u)=v(u, u)$ is the Airy stress function as defined in (2.3).

In what follows below, we will often make use of the above theorem to give

$$
<\left[u, F_{0}\right], u>+\|u\|^{2} \leq \delta \Pi_{*}(u)+C_{\delta, F_{0}} .
$$

We now compute $\frac{d}{d t} V\left(S_{t} y\right)$ :

$$
\begin{aligned}
\frac{d}{d t} V\left(S_{t} y\right)= & \frac{d}{d t} \mathscr{E}(t)+\nu<u_{t t}, u>+\nu\left\|u_{t}\right\|^{2}+k \nu<u_{t}, u> \\
& -<q^{u}, u_{t}>-<q_{t}^{u}, u> \\
& +\mu \frac{d}{d t}\left\{\int_{t-t^{*}}^{t} \Pi_{*}\left(u(s) d s+\int_{0}^{t^{*}} \int_{t-s}^{t} \Pi_{*}(u(\tau)) d \tau d s\right\}\right. \\
= & -k\left\|u_{t}\right\|^{2}+<p_{0}-L u, u_{t}>-<q_{t}^{u}, u> \\
& +\nu<u_{t t}+k u_{t}, u>+\nu\left\|u_{t}\right\|^{2}+\mu \Pi_{*}(u(t))-\mu \Pi\left(u\left(t-t^{*}\right)\right) \\
& +\mu t^{*} \Pi_{*}(u(t))-\mu \int_{-t^{*}}^{0} \Pi_{*}(u(t-\tau)) d \tau
\end{aligned}
$$

Now, we make use of the relation

$$
u_{t t}+k u_{t}=-\Delta^{2} u+p_{0}+q^{u}(t)+\left[u, v(u)+F_{0}\right]-L u
$$

owing to (2.14). Substituting this back into the relation above and simplifying yields:

$$
\begin{aligned}
\frac{d}{d t} V\left(S_{t} y\right)= & -(k-\nu)\left\|u_{t}\right\|^{2}-\nu\|\Delta u\|^{2}-\nu\|\Delta v(u)\|^{2}-\mu \Pi_{*}\left(u\left(t-t^{*}\right)\right)-\mu \int_{-t^{*}}^{0} \Pi_{*}(u(t+\tau)) d \tau \\
& +\nu<\left[u, F_{0}\right], u>+\nu<q^{u}, u>+<p_{0}-L u, u_{t}>+\nu<p_{0}-L u, u> \\
& -<q_{t}^{u}, u>+\mu\left(t^{*}+1\right) \Pi_{*}(u(t)) .
\end{aligned}
$$


In the estimate that follows we make use of (a) standard splitting via Young's inequality, (b) the bound in Proposition 4.7. (c) the assumption that $0<\nu<\min \{1, k\}$. Then we have for all $\epsilon>0$

$$
\begin{aligned}
\frac{d}{d t} V\left(S_{t} y\right) \leq & (-k+\nu)\left\|u_{t}\right\|^{2}+\left(\frac{\mu\left(1+t^{*}\right)}{2}-\nu\right)\|\Delta u\|^{2}+\left(\frac{\mu\left(1+t^{*}\right)}{4}-\nu\right)\|\Delta v(u)\|^{2} \\
& -\mu \Pi\left(u\left(t-t^{*}\right)\right)-\mu \int_{-t^{*}}^{0} \Pi_{*}(u(t+\tau)) d \tau \\
& +\epsilon\left\|u_{t}\right\|^{2}+\epsilon\left(\|\Delta u\|^{2}+\|\Delta v(u)\|^{2}\right)+C_{\epsilon, \delta, p_{0}, F_{0}}+\epsilon\left\|q^{u}\right\|^{2}+\left|<q_{t}^{u}, u>\right| .
\end{aligned}
$$

Now, using (2.16):

$$
\left\|q^{u}(t)\right\|^{2} \leq C \int_{t-t^{*}}^{t}\|\Delta u(\tau)\|^{2} d \tau
$$

and (2.17) with $\psi=u$ :

$$
\left|<q_{t}^{u}(t), u(t)>\right| \leq \epsilon\left[\|\Delta u(t)\|^{2}+\left\|\Delta u\left(t-t^{*}\right)\right\|^{2}+\int_{-t^{*}}^{0}\|\Delta u(t+\tau)\|^{2} d \tau\right]+C_{\epsilon, t^{*}}\|u(t)\|^{2}
$$

we have (again, making use of Proposition 4.7) the following inequality for all $\epsilon>0$ :

$$
\begin{aligned}
\frac{d}{d t} V\left(S_{t} y\right) \leq & (-k+\nu+\epsilon)\left\|u_{t}\right\|^{2}+\left(\frac{\mu\left(1+t^{*}\right)}{2}+2 \epsilon-\nu\right)\|\Delta u\|^{2}+\left(\frac{\mu\left(1+t^{*}\right)}{4}+\epsilon-\nu\right)\|\Delta v(u)\|^{2} \\
& -(\mu-2 \epsilon) \Pi_{*}\left(t-t^{*}\right)-(\mu-4 \epsilon) \int_{-t^{*}}^{0} \Pi_{*}(u(t+\tau)) d \tau+C_{\epsilon}\|u(t)\|^{2}+C_{\epsilon, \mu, p_{0}, F_{0}} .
\end{aligned}
$$

And, for $0<\nu<k$, and for $\mu$ and $\epsilon$ sufficiently small, we have the following lemma:

Lemma 4.8. For all $k>0$ there exist $\mu, \nu>0$ and $c\left(\mu, \nu, t^{*}, k\right)>0$ and $C\left(\mu, \nu, p_{0}, F_{0}\right)>0$ such that

$$
\frac{d}{d t} V\left(S_{t} y\right) \leq-c\left\{\left\|u_{t}\right\|^{2}+\|\Delta u\|^{2}+\|\Delta v(u)\|^{2}+\Pi_{*}\left(u\left(t-t^{*}\right)\right)+\int_{-t^{*}}^{0} \Pi_{*}(u(t+\tau)) d \tau\right\}+C .
$$

From this lemma and the upper bound in (4.8), we have for some $\beta>0$ sufficiently small (again, depending on $\mu$ and $\nu$ ):

$$
\frac{d}{d t} V\left(S_{t} y\right)+\beta V\left(S_{t} y\right) \leq C, \quad t>0,
$$

The estimate above in (4.9) implies (by a version of Gronwall's inequality) that

$$
V\left(S_{t} y\right) \leq V(y) e^{-\beta t}+\frac{C}{\beta}\left(1-e^{-\beta t}\right) .
$$

Hence, the set

$$
\mathscr{B} \equiv\left\{y \in \mathbf{H}: V(y) \leq 1+\frac{C}{\beta}\right\},
$$

is a bounded forward invariant absorbing set. This gives that $\left(\mathbf{H}, S_{t}\right)$ is dissipative. 


\subsection{Asymptotic Smoothness}

Recall that our dynamical system is $\left(S_{t}, \mathbf{H}\right)$, where $S_{t}$ is the evolution operator corresponding to plate solutions to (2.14) and $\mathbf{H}=H_{0}^{2}(\Omega) \times L_{2}(\Omega) \times L_{2}\left(-t^{*}, 0 ; H_{0}^{2}(\Omega)\right)$. To show asymptotic smoothness of this dynamical system, we will make use of an abstract Theorem 4.2 .

To make use of this theorem, we will consider our functional $\Psi$ to be comprised of lower order terms (compact with respect to $\mathbf{H})$ and quasicompact $\left(\int_{s}^{t}<f\left(u^{1}\right)-f\left(u^{2}\right), z_{t}>d \tau\right)$ terms. On the LHS of the above estimate, we see that we need to produce an estimate which bounds trajectories in $\mathbf{H}$, i.e. $\left\|\left(u(t), u_{t}(t), u^{t}\right)\right\|_{\mathbf{H}}^{2}$ (taking the metric $d$ to be $\|\cdot\|_{\mathbf{H}}$ ). Such an estimate will be produced below by combining our energy estimates produced earlier:

Lemma 4.9. Suppose $z=u^{1}-u^{2}$ is as in (2.26), with $y^{i}(t)=\left(u^{i}(t), u_{t}(t)^{i}, u^{t, i}\right)$ and $y^{i}(t) \in \mathscr{B}_{R}(\mathbf{H})$ for all $t \geq 0$. Also, let $\eta>0$ and $E_{z}(t)$ be defined as in 2.27). Then for every $0<\epsilon<1$ there exists $T=T_{\epsilon}(R)$ such that the following estimate

$$
E_{z}(T)+\int_{T-t^{*}}^{T}\|z(\tau)\|_{2}^{2} d \tau \leq \epsilon+\Psi_{\epsilon, T, R}\left(y^{1}, y^{2}\right)
$$

holds, where

$$
\begin{aligned}
\Psi_{\epsilon, T, R}\left(y^{1}, y^{2}\right) \equiv & C(R, T) \sup _{\tau \in[0, T]}|| z(\tau) \|_{2-\eta}^{2}+a_{1}\left|\int_{0}^{T}<f\left(u^{1}(\tau)\right)-f\left(u^{2}(\tau)\right), z_{t}(\tau)>d \tau\right| \\
& +a_{2}\left|\int_{0}^{T} \int_{s}^{T}<f\left(u^{1}(\tau)\right)-f\left(u^{2}(\tau)\right), z_{t}(\tau)>d \tau d s\right| .
\end{aligned}
$$

Proof. It follows from (4.6) by dividing by $T$ and taking $T$ large enough.

In Lemma 4.9 above, we have obtained the necessary estimate for asymptotic smoothness; it now suffices to show that $\Psi$, as defined above, has the compensated compactness condition described in Theorem 4.2 .

Before proceeding, let us introduce some notation which will be used throughout the remainder of this section and in the following section. We will write

$$
\text { l.o.t. }=\sup _{\tau \in[0, T]}\|z(\tau)\|_{2-\eta}^{2}, \quad \mathcal{F}(z)=f\left(u^{1}\right)-f\left(u^{2}\right) .
$$

Theorem 4.10. The dynamical system $\left(S_{t}, \mathbf{H}\right)$ generated by weak solutions to 2.14) is asymptotically smooth.

Proof. In line with the discussion above, we aim to make use of Theorem 4.2. To do so, it suffices to show the compensated compactness condition for $\Psi_{\epsilon, T, R}$ which we now write as $\Psi$, with $\epsilon, T$, and $R$ fixed along with the other constants given by the equation. Let $B$ be a bounded, positively invariant set in $\mathbf{H}$, and let $\left\{y_{n}\right\} \subset B \subset \mathscr{B}_{R}(\mathbf{H})$. We would like to show that

$$
\liminf _{m} \liminf _{n} \Psi\left(y_{n}, y_{m}\right)=0 .
$$

More specifically, for any initial data $U_{0}^{1}=\left(u_{0}^{1}, u_{1}^{1}, \eta^{1}\right), U_{0}^{2}=\left(u_{0}^{2}, u_{1}^{2}, \eta^{2}\right) \in B$ (where $\eta^{i}$ belongs to 
$\left.L_{2}\left(-t^{*}, 0: H_{0}^{2}(\Omega)\right)\right)$ we define

$$
\widetilde{\Psi}^{2}\left(U_{0}^{1}, U_{0}^{2}\right)=\left|\int_{0}^{T}<\mathcal{F}(z)(\tau), z_{t}(\tau)>d \tau\right|+\left|\int_{0}^{T} \int_{s}^{T}<\mathcal{F}(z(\tau)), z_{t}(\tau)>d \tau d s\right|
$$

where the function $z=u^{1}-u^{2}$ has initial data $U_{0}^{1}-U_{0}^{2}$ and solves (2.26). The key to compensated compactness is the following representation for the bracket [14, pp. 598-599]:

$$
\begin{aligned}
<\mathcal{F}(z)(\tau), z_{t}(\tau)>= & \frac{1}{4} \frac{d}{d \tau}\left\{-\left\|\Delta v\left(u^{1}\right)\right\|^{2}-\left\|\Delta v\left(u^{2}\right)\right\|^{2}+2<[z, z], F_{0}>\right\} \\
& -<\left[v\left(u^{2}\right), u^{2}\right], u_{t}^{1}>-<\left[v\left(u^{1}\right), u^{1}\right], u_{t}^{2}>.
\end{aligned}
$$

Integrating the above expression in time and evaluating on the difference of two solutions $z^{n, m}=$ $w^{n}-w^{m}$ with initial data $W_{0}^{n}-W_{0}^{m}$, where $w^{i} \rightarrow w$, yields:

$$
\begin{aligned}
\lim _{n \rightarrow \infty} \lim _{m \rightarrow \infty} \int_{s}^{T} & <\mathcal{F}\left(z^{n, m}\right)(\tau), z_{t}^{n, m}(\tau)>d \tau=\frac{1}{2}\left\{\|\Delta v(w(s))\|^{2}-\|\Delta v(w(T))\|^{2}\right\} \\
& -\lim _{n \rightarrow \infty} \lim _{m \rightarrow \infty} \int_{s}^{T}\left\{<\left[v\left(w^{n}\right), w^{n}\right], w_{t}^{m}>+<\left[v\left(w^{m}\right), w^{m}\right], w_{t}^{n}>\right\},
\end{aligned}
$$

where we have used (i) the weak convergence in $H^{2}(\Omega)$ of $z^{n, m}$ to 0 , and (ii) compactness of $\Delta v(\cdot)$ from $H^{2}(\Omega) \rightarrow L_{2}(\Omega)$ as in Lemma 2.11. The iterated limit in (4.11) is handled via iterated weak convergence, as follows:

$$
\begin{aligned}
& \lim _{n \rightarrow \infty} \lim _{m \rightarrow \infty} \int_{s}^{T}\left\{<\left[v\left(w^{n}\right), w^{n}\right], w_{t}^{m}>+<\left[v\left(w^{m}\right), w^{m}\right], w_{t}^{n}>\right\} \\
& =2 \int_{s}^{T}<[v(w), w], w_{t}>=\frac{1}{2}\|\Delta v(w)(s)\|^{2}-\frac{1}{2}\|\Delta v(w)(T)\|^{2} .
\end{aligned}
$$

This yields the desired conclusion, that

$$
\lim _{n \rightarrow \infty} \lim _{m \rightarrow \infty} \int_{s}^{T}\left(\mathcal{F}<z^{n, m}(\tau)\right), z_{t}^{n, m}(\tau)>d \tau=0
$$

The second integral term in $\widetilde{\Psi}^{2}$ is handled similarly. Since the term l.o.t. above is compact (below energy level) via the Sobolev embedding, as a consequence we obtain

$$
\liminf _{m \rightarrow \infty} \liminf _{n \rightarrow \infty} \widetilde{\Psi}\left(y_{n}, y_{m}\right)=0
$$

This concludes the proof of the asymptotic smoothness via Theorem 4.2

Having shown the asymptotic smoothness property, we can now conclude by Theorem 4.1 that there exists a compact global attractor $\mathbf{A} \subset \mathbf{H}$ for the dynamical system $\left(S_{t}, \mathbf{H}\right)$. 


\subsection{Quasistability Estimate}

In this section we refine our methods in the asymptotic smoothness calculation and work on trajectories from the attractor, whose existence has been established in the previous sections.

Analyzing (4.6), we may also write

$$
\begin{gathered}
T\left[E_{z}(T)+\int_{T-t^{*}}^{T} E_{z}(\tau) d \tau\right] \leq c\left(E_{z}(0)+\int_{-t^{*}}^{0}\|z(\tau)\|_{2}^{2} d \tau\right) \\
+C \cdot T \sup _{s \in[0, T]}\left|\int_{s}^{T}<\mathcal{F}(z), z_{t}>d \tau\right|+C(R, T) \sup _{\tau \in[s, t]}\|z\|_{2-\eta}^{2},
\end{gathered}
$$

where $\mathcal{F}(z)$ is given in (4.10). We note that $c$ does not depend on $T \geq \min \left\{1,2 t^{*}\right\}$, and l.o.t. is of quadratic order.

In order to prove the quasistability estimate (as in (4.1)), we have to handle the non-compact term $<\mathcal{F}(z), z_{t}>$. We recall the relation (4.4) in Theorem 4.4 if $u^{i} \in C\left(s, t ; H^{2}(\Omega)\right) \cap C^{1}\left(s, t ; L_{2}(\Omega)\right)$ with $u^{i}(\tau) \in \mathscr{B}_{R}\left(L_{2}(\Omega)\right)$ for $\tau \in[s, t]$, then

$$
\left|\int_{s}^{t}<\mathcal{F}(z), z_{t}(\tau)>d \tau \leq C(R) \sup _{\tau \in[s, t]}\right|\left|z \|_{2-\eta}^{2}+C \frac{1}{2}\right| \int_{s}^{t} P(z(\tau)) d \tau \mid
$$

for some $0<\eta<1 / 2$. Here $P(z)$ is given by (4.3).

Let $\gamma_{u^{1}}=\left\{\left(u^{1}(t), u_{t}^{1}(t),\left[u^{1}\right]^{t}\right): t \in \mathbb{R}\right\}$ and $\gamma_{u^{2}}=\left\{\left(u^{2}(t), u_{t}^{2}(t),\left[u^{2}\right]^{t}\right): t \in \mathbb{R}\right\}$ be trajectories from the attractor $\mathbf{A}$. It is clear that for the pair $u^{1}(t)$ and $u^{2}(t)$ satisfy the hypotheses of the estimate in (4.13) for every interval $[s, t]$. Our main goal is to handle the second term on the right hand side of (4.13) which is of critical regularity. To accomplish this we shall use the already established compactness of the attractor in the state space $\mathbf{H}=H_{0}^{2}(\Omega) \times L_{2}(\Omega) \times L_{2}\left(-t^{*}, 0 ; H_{0}^{2}(\Omega)\right)$.

Since for every $\tau \in \mathbb{R}$, the element $u_{t}^{i}(\tau)$ belongs to a compact set in $L_{2}(\Omega)$, by density of $H_{0}^{2}(\Omega)$ in $L_{2}(\Omega)$ we can assume, without a loss of generality, that for every $\epsilon>0$ there exists a finite set $\left\{\phi_{j}\right\} \subset H_{0}^{2}(\Omega), j=1,2, \ldots, n(\epsilon)$, such that for all $\tau \in \mathbb{R}$ we can find indices $j_{1}(\tau)$ and $j_{2}(\tau)$ so that

$$
\left\|u_{t}^{1}(\tau)-\phi_{j_{1}(\tau)}\right\|+\left\|u_{t}^{2}(\tau)-\phi_{j_{2}(\tau)}\right\| \leq \epsilon \text { for all } \tau \in \mathbb{R} .
$$

Let $P(z)$ be given by (4.3) with the pair $u^{1}(t)$ and $u^{2}(t)$ and

$$
P_{j_{1}, j_{2}}(z) \equiv-\left(\phi_{j_{1}},\left[u^{1}, v(z)\right]\right)-\left(\phi_{j_{2}},\left[u^{2}, v(z, z)\right]\right)-\left(\phi_{j_{1}}+\phi_{j_{2}},\left[z, v\left(u^{1}+u^{2}, z\right)\right]\right),
$$

where $z(t)=u^{1}(t)-u^{2}(t)$. It can be easily shown that for all $j_{1}, j_{2} \leq n(\epsilon)$

$$
\left\|P(z(\tau))-P_{j_{1}(\tau), j_{2}(\tau)}(z(\tau))\right\| \leq \epsilon C(\mathbf{A})\|z(\tau)\|_{2}^{2}
$$

uniformly in $\tau \in \mathbb{R}$.

Starting with the estimate (1.4.17) page 41 [14,

$$
\|[u, w]\|_{-2} \leq C\|u\|_{2-\beta}\|w\|_{1+\beta}, \quad \forall \beta \in[0,1)
$$


and exploiting elliptic regularity one obtains

$$
\|[u, v(z, w)]\|_{-2} \leq C|| u\left\|_{2-\beta}\right\|[z, w] \mid\left\|_{-2} \leq C\right\| u\left\|_{2-\beta}\right\| z\left\|_{2-\beta_{1}}\right\| w \|_{1+\beta_{1}}
$$

where above inequality holds for any $\beta, \beta_{1} \in[0,1)$

Recalling the additional smoothness of $\phi_{j} \in H_{0}^{2}(\Omega)$, along with the estimate in (4.15) applied with $\beta=\beta_{1}=\eta$, and accounting the structure of $P_{j}$ terms one obtains:

$$
\left\|P_{j_{1}, j_{2}}(z)\right\| \leq C(\mathbf{A})\left(\left\|\phi_{j_{1}}\right\|_{2}+\left\|\phi_{j_{2}}\right\|_{2}\right)\|z(\tau)\|_{2-\eta}^{2}
$$

for some $0<\eta<1$. So we have

$$
\sup _{j_{1}, j_{2}}\left\|P_{j_{1}, j_{2}}(z)\right\| \leq C(\epsilon)\|z(\tau)\|_{2-\eta}^{2} \quad \text { for some } 0<\eta<1,
$$

where $C(\epsilon) \rightarrow \infty$ when $\epsilon \rightarrow 0$. Taking into account (4.14) and (4.16) in (4.13) we obtain

$$
\left|\int_{s}^{t}<\mathcal{F}(z), z_{t}>\right| \leq C(\epsilon, T, \mathbf{A}) \sup _{\tau \in[s, t]}\|z(\tau)\|_{2-\eta}^{2}+\epsilon \int_{s}^{t}\|z(\tau)\|_{2}^{2} d \tau
$$

for all $s \in \mathbb{R}$ with $\eta>0$ and $t>s$. Considering (4.17) and taking $T$ sufficiently large, we have from (4.12)

$$
E_{z}(T)+\int_{T-t^{*}}^{T}\|z(\tau)\|_{2}^{2} d \tau \leq \gamma\left(E_{z}(0)+\int_{-t^{*}}^{0}\|z(\tau)\|_{2}^{2} d \tau\right)+C\left(\mathbf{A}, T, k, t^{*}\right) \sup _{\tau \in[0, T]}\|z(\tau)\|_{2-\eta}^{2}
$$

with $\gamma<1$. Thus by the standard argument (see [14) we finally conclude that for $y(t)=\left(z(t), z_{t}(t), z^{t}\right)$

$$
\|y(t)\|_{\mathbf{H}}^{2} \leq C(\sigma, \mathbf{A})\|y(0)\|_{\mathbf{H}}^{2} e^{-\sigma t}+C \sup _{\tau \in[0, t]}\|z(\tau)\|_{2-\eta}^{2} .
$$

Hence on the strength of Theorem 4.3 , applied with $B=\mathbf{A}$ and

$$
\mathbf{H}=H_{0}^{2}(\Omega) \times L_{2}(\Omega) \times L_{2}\left(-t^{*}, 0 ; H_{0}^{2}(\Omega)\right)
$$

where $H_{1}=H^{2-\eta}(\Omega)$, we conclude that $\mathbf{A}$ has a finite fractal dimension.

Additionally, Theorem 4.3 guarantees that

$$
\left\|u_{t t}(t)\right\|^{2}+\left\|u_{t}(t)\right\|_{2}^{2} \leq C \text { for all } t \in \mathbb{R} .
$$

Since $u_{t} \in H^{2}(\Omega) \subset C(\Omega)$, elliptic regularity theory for

$$
\Delta^{2} u=-u_{t t}-k u_{t}-f(u)-L u+q\left(u^{t}, t\right)
$$

with the clamped boundary conditions give that

$$
\|u(t)\|_{4}^{2} \leq C \text { for all } t \in \mathbb{R} .
$$


Thus, we can conclude additional regularity of the trajectories from the attractor $\mathbf{A} \subset \mathbf{H}$ stated in Theorem 3.1 .

We have now completed the proof of Theorem 3.1

\subsection{Extensions and Open Problems}

In this section, we briefly mention possible extensions of our results and open problems pertaining to the models discussed above.

- Other homogeneous boundary conditions: hinged, simply supported, free-type, or combinations thereof.

- Nonlinear internal damping, i.e. considering $k\left(u_{t}\right)$ in the plate equation, where $k(\cdot)$ is a Nemitsky operator.

- Boundary damping via (nonlinear) hinged dissipation (31).

- Other physical nonlinearities; replacing the von Karman nonlinearity in the considerations above with Berger or Kirchoff-type nonlinearity (as discussed in [7, 9, 17]). This should not present major technical issues.

- Convergence to equilibria; one may conjecture that individual trajectories of the full flow-plate system converge to single elements of the attractor. However, Dowell's conjecture (supported by numerics) states that only in the subsonic case solutions stabilize to stationary points, and in the supersonic case, locally stable periodic (or even chaotic) orbits are possible. Hence, it is likely that one can discuss this convergence only in the subsonic case. The principal issue here is finiteness of the dissipation integral

$$
\int_{0}^{\infty}\left\|u_{t}\right\|^{2} d t
$$

We can easily guarantee this if we have additional plate damping in the coupled system. In this case we can achieve stabilization in the same way as it done in 14 for the rotational case (see also [10]). So the issue becomes how to obtain some form of finiteness of dissipation integral in the case where full energy of coupled system is preserved (the reduction result Theorem 2.3 is not employed). This issue remains open (see also the corresponding remark in [14, Section 12.4.2]). 


\section{$5 \quad$ Flow-Plate Interactions}

In this section we provide sketch of the proof of Theorem 2.3. which is needed for our principal application of Theorem 3.1 to the long-time dynamics of the fully coupled model:

$$
\begin{cases}u_{t t}+\Delta^{2} u+k u_{t}+f(u)=p_{0}+\left(\partial_{t}+U \partial_{x}\right) \gamma[\phi] & \text { in } \Omega \times(0, T), \\ u(0)=u_{0} ; u_{t}(0)=u_{1}, & \\ u=\partial_{\nu} u=0 & \text { on } \partial \Omega \times(0, T), \\ \left(\partial_{t}+U \partial_{x}\right)^{2} \phi=\Delta \phi & \text { in } \mathbb{R}_{+}^{3} \times(0, T), \\ \phi(0)=\phi_{0} ; \quad \phi_{t}(0)=\phi_{1}, & \text { on } \mathbb{R}_{\{(x, y)\}}^{2} \times(0, T) .\end{cases}
$$

Proof. By Theorem 2.1] the system (5.1) generates a strongly continuous semigroup $T_{t}$ on $H$. This is to say that $\left(\phi(t), \phi_{t}(t), u(t), u_{t}(t)\right)=T_{t}\left(\phi_{0}, \phi_{1}, u_{0}, u_{1}\right), t>0$. The main idea behind the proof of Theorem 2.3 (see [14, Section 6.6.5]) is to split gas flow variable $\phi$ in two components: $\phi(\mathbf{x}, t)=$ $\phi^{*}(\mathbf{x}, t)+\phi^{* *}(\mathbf{x}, t)$, where $\phi^{*}(\mathbf{x}, t)$ solves (2.4) with $d(\mathbf{x}, t) \equiv 0$ and $\phi^{* *}(\mathbf{x}, t)$ is solution to nonhomogenous problem (2.4) with the zero initial data $\phi_{0}=0$ and $\phi_{1}=0$. Here we note that with $\left(\phi_{0}, \phi_{1}\right) \in H^{1}\left(\mathbb{R}_{+}^{3}\right) \times L_{2}\left(\mathbb{R}_{+}^{3}\right)$ one obtains [32, 16] $\left(\phi^{*}(t), \phi_{t}^{*}(t)\right) \in H^{1}\left(\mathbb{R}_{+}^{3}\right) \times L_{2}\left(\mathbb{R}_{+}^{3}\right)$. Thus, by Theorem 2.1 we also have that

$$
\left(\phi^{* *}(t), \phi_{t}^{* *}(t) \in H^{1}\left(\mathbb{R}_{+}^{3}\right) \times L_{2}\left(\mathbb{R}_{+}^{3}\right) .\right.
$$

Note that this last property is not valid for a flow solution with $L_{2}$ boundary Neumann datd 4 . However, the improved regularity is due to the interaction with the plate and the resulting cancelations on the interface. Moreover, we also obtain a meaningful "hidden trace regularity" for the aeroelastic potential on the boundary of the structure [16]:

$$
\left(\partial_{t}+U \partial_{x}\right) \gamma[\phi] \in L_{2}\left(0, T ; H^{-1 / 2}(\Omega)\right)
$$

where $T$ is arbitrary.

Then, using the Kirchhoff type representation for the solution $\phi^{*}(\mathbf{x}, t)$ in $\mathbb{R}_{+}^{3}$ (see, e.g., Theorem 6.6.12 in [14]), we can conclude that if the initial data $\phi_{0}$ and $\phi_{1}$ are localized in the ball $K_{R}=\left\{\mathbf{x} \in \mathbb{R}_{+}^{3}:|\mathbf{x}| \leq R\right\}$, then by finite dependence on the domain of the signal in three dimensions (Hyugen's principle), one obtains $\phi^{*}(\mathbf{x}, t) \equiv 0$ for all $\mathbf{x} \in K_{R}$ and $t \geq t_{R}$. Thus we have that

$$
\left(\partial_{t}+U \partial_{x}\right) \gamma\left[\phi^{*}\right] \equiv 0, \quad \mathbf{x} \in \Omega, t \geq t_{R} .
$$

Thus it remains to consider flow variable $\phi^{* *}$, whose aeroelastic potential on the boundary coincides with that of $\phi$, and hence it displays regularity as in (5.2) . This allows one to perform calculations as

\footnotetext{
${ }^{4}$ The general theory will provide at most $H^{2} / 3\left(\mathbb{R}_{+}^{3} \times[0, T]\right)$.
} 
in [14, Theorem 6.6.10]) in order to obtain the representation

$$
\begin{aligned}
\left(\partial_{t}+U \partial_{x}\right) \gamma[\phi]= & -d(x, y, t) \\
& +\frac{1}{2 \pi} \int_{0}^{t} d s \int_{0}^{2 \pi} d \theta\left[M_{\theta} d\right](x-(U+\sin \theta) s, y-s \cos \theta, t-s) .
\end{aligned}
$$

Now using the same calculations as in [14, p.333] we arrive at the following equation:

$$
u_{t t}+\Delta^{2} u+k u_{t}-\left[u, v(u)+F_{0}\right]=p_{0}-\left(\partial_{t}+U \partial_{x}\right) u-q^{u}(t)
$$

for $t$ large enough, with

$$
q^{u}(t)=\frac{1}{2 \pi} \int_{0}^{t^{*}} d s \int_{0}^{2 \pi} d \theta\left[M_{\theta}^{2} \widehat{u}\right](x-(U+\sin \theta) s, y-s \cos \theta, t-s) .
$$

Here, $\widehat{u}$ is the extension of $u$ by 0 outside of $\Omega ; M_{\theta}=\sin \theta \partial_{x}+\cos \theta \partial_{y}$ and $t^{*}$ is given by (2.9).

\section{Appendix: Properties of delayed force $q$}

In this Appendix we consider properties of the delayed (aerodynamic type) force and prove Proposition 2.4 and Lemma 2.12 .

\subsection{Hidden Compactness of Retarded Potential: Proof of Proposition 2.4}

The proof of the bounds (2.10) -(2.12) can be found in 8 and 14 . Thus we need to check (2.13) only. Without loss of generality we can assume $u \in C\left(-t^{*},+\infty ; C_{0}^{\infty}(\Omega)\right)$.

For any $\psi \in H_{0}^{1}(\Omega)$ we have

$$
\begin{aligned}
<q_{t}^{u}(t), \psi>= & \left\langle\int_{0}^{2 \pi} \frac{1}{2 \pi}\left[M_{\theta}^{2} \widehat{u}\right](\mathbf{x}(U, \theta, 0), t) d \theta, \psi\right\rangle \\
& -\left\langle\int_{0}^{2 \pi} \frac{1}{2 \pi}\left[M_{\theta}^{2} \widehat{u}\right]\left(\mathbf{x}\left(U, \theta, t^{*}\right), t-t^{*}\right) d \theta, \psi\right\rangle \\
& +\left\langle\left(\int_{0}^{t^{*}} \int_{0}^{2 \pi}(U+\sin \theta) \frac{1}{2 \pi}\left[M_{\theta}^{2} \widehat{u}\right]_{x}(\mathbf{x}(U, \theta, s), t-s) d \theta d s\right), \psi\right\rangle \\
& +\left\langle\left(\int_{0}^{t^{*}} \int_{0}^{2 \pi}(\cos \theta) \frac{1}{2 \pi}\left[M_{\theta}^{2} \widehat{u}\right]_{y}(\mathbf{x}(U, \theta, s), t-s) d \theta d s\right), \psi\right\rangle,
\end{aligned}
$$

recalling that $\mathbf{x}(U, \theta, s)=(x-(U+\sin \theta) s, y-s \cos \theta)$. In all integrals above we extend the integration 
over $\Omega$ to all of $\mathbb{R}^{2}$ and change spatial variables.

$$
\begin{aligned}
\left|<q_{t}^{u}(t), \psi>\right| \leq & C\left\{\left|\int_{0}^{2 \pi} \int_{\mathbb{R}^{2}}\left[M_{\theta}^{2} \widehat{u}\right](\tau) \psi d \mathbf{x} d \theta\right|\right. \\
& +\left|\int_{0}^{2 \pi} \int_{\mathbb{R}^{2}}\left[M_{\theta}^{2} \widehat{u}\right]\left(t-t^{*}\right) \psi\left(\mathbf{x}\left(U, \theta,-t^{*}\right)\right) d \mathbf{x} d \theta\right| \\
& +\left|\int_{0}^{t^{*}} \int_{0}^{2 \pi} \int_{\mathbb{R}^{2}}(U+\sin \theta)\left[M_{\theta}^{2} \widehat{u}\right]_{x}(\mathbf{x}, \tau-s) \psi(\mathbf{x}(U, \theta,-s)) d \mathbf{x} d \theta d s\right| \\
& \left.+\left|\int_{0}^{t^{*}} \int_{0}^{2 \pi} \int_{\mathbb{R}^{2}} \cos \theta\left[M_{\theta}^{2} \widehat{u}\right]_{y}(\mathbf{x}, \tau-s) \psi(\mathbf{x}(U, \theta,-s)) d \mathbf{x} d \theta d s\right|\right\} .
\end{aligned}
$$

Now, we note that $M_{\theta}$ can be moved under the integration in $\mathbf{x}$, and we have $\left|M_{\theta} f(\mathbf{x})\right| \leq\left|f_{x}(\mathbf{x})\right|+$ $\left|f_{y}(\mathbf{x})\right|$ for all $f$. Hence, we integrate by parts with a single $M_{\theta}$ in both of the first integrals; in the third and fourth integrals, we also integrate by parts once as well. This leaves us with:

$$
\left|<q_{t}^{u}(t), \psi>\right| \leq C\left\{\|u(t)\|_{1}+\left\|u\left(t-t^{*}\right)\right\|_{1}+\int_{-t^{*}}^{0}\|u(t+\tau)\|_{2} d \tau\right\}\|\psi\|_{1} .
$$

This implies the conclusion in (2.13). The proof of Proposition 2.4 is complete.

\subsection{Proof of Lemma 2.12}

The relation in (2.21) easily follows from (2.15) and simple formula:

$$
\int_{0}^{t} d \tau \int_{\tau-t *}^{\tau} \phi(s) d s \leq t^{*} \int_{-t *}^{t} \phi(s) d s, \quad \forall \phi \in L_{1}(0, T) .
$$

Now we prove (2.22). Integrating by parts in $t$, and then applying (2.17) with $\psi=u(t)$, we have:

$$
\begin{aligned}
\left|\int_{0}^{t}<q^{u}(\tau), u_{t}(\tau)>d \tau\right| \leq & C\left\{\int_{0}^{t}\|u(\tau)\|_{2}\|u(\tau)\|_{2-\eta} d \tau+\int_{0}^{t}\left\|u\left(\tau-t^{*}\right)\right\|_{2}\|u(\tau)\|_{2-\eta} d \tau\right. \\
& +\int_{0}^{t} \int_{0}^{t^{*}}\|u(\tau-s)\|_{2}\|u(\tau)\|_{2-\eta} d s d \tau \\
& \left.+\left\|q^{u}(t)\right\|_{-\sigma}\|u(t)\|_{\sigma}+\left\|q^{u}(0)\right\|_{-\sigma}\|u(0)\|_{\sigma}\right\} \\
\leq & \epsilon \int_{-t^{*}}^{t}\|u(\tau)\|_{2}^{2}+C_{\epsilon} T \sup _{0, t}\|u(\tau)\|_{2-\eta}^{2} \\
& +\epsilon \int_{0}^{t} \int_{0}^{t^{*}}\|u(\tau-s)\|_{2}^{2} d s d \tau+C_{\epsilon} t^{*} \int_{0}^{t}\|u(\tau)\|_{2-\eta}^{2} d \tau \\
& +C\left\{\epsilon\left\|q^{u}(t)\right\|_{-\sigma}^{2}+\frac{1}{\epsilon}\|u(t)\|_{\sigma}^{2}+\epsilon\left\|q^{u}(0)\right\|_{-\sigma}^{2}+\frac{1}{\epsilon}\|u(0)\|_{\sigma}^{2}\right\}
\end{aligned}
$$


After rescaling of $\epsilon$ this implies

$$
\begin{aligned}
\left|\int_{0}^{t}<q^{u}(\tau), u_{t}(\tau)>d \tau\right| \leq & C\left(t^{*}, \epsilon\right)(1+T) \sup _{\tau \in[0, t]}\|u(\tau)\|_{2-\eta}^{2}+\epsilon \int_{-t^{*}}^{t}\|u(\rho)\|_{2}^{2} d \rho d \tau \\
& +\epsilon\left\{\left\|q^{u}(t)\right\|_{-\sigma}^{2}+\left\|q^{u}(0)\right\|_{-\sigma}^{2}\right\} .
\end{aligned}
$$

Therefore by (2.16) this implies (2.22) with $\eta_{*}=\min \{\eta, 2-\sigma\}$.

\section{References}

[1] A. Babin and M. Vishik, Attractors of Evolution Equations, North-Holland, Amsterdam, 1992.

[2] A. V. Balakrishnan, Aeroelasticity-Continuum Theory. Springer Verlag, 2012.

[3] V.V. Bolotin, Nonconservative problems of elastic stability. Pergamon Press, Oxford, 1963.

[4] A. Boutet de Monvel and I. Chueshov, The problem of interaction of von Karman plate with subsonic flow gas, Math. Methods in Appl. Sc., 22 (1999), 801-810.

[5] L. Boutet de Monvel and I. Chueshov, Non-linear oscillations of a plate in a flow of gas, C.R. Acad. Sci. Paris, Ser.I, 322 (1996), 1001-1006.

[6] L. Boutet de Monvel and I. Chueshov, Oscillation of von Karman's plate in a potential flow of gas, Izvestiya RAN: Ser. Mat. 63 (1999), 219-244.

[7] L. Boutet de Monvel, I. Chueshov, and A. Rezounenko, Long-time behaviour of strong solutions of retarded nonlinear PDEs, Comm. PDEs, 22 (1997), 1453-1474.

[8] I. Chueshov, On a certain system of equations with delay, occurring in aeroelasticity, Teor. Funktsii Funktsional. Anal. i Prilozhen. No. 54 (1990), 123-130 (in Russian); translation in J. Soviet Math., 58 (1992), 385-390.

[9] I. Chueshov, Introduction to the Theory of Infinite-Dimensional Dissipative Systems, Acta, Kharkov, 1999, in Russian; English translation: Acta, Kharkov, 2002;

see also http://www.emis.de/monographs/Chueshov/

[10] I. Chueshov, Dynamics of von Karman plate in a potential flow of gas: rigorous results and unsolved problems, Proceedings of the 16th IMACS World Congress, Lausanne (Switzerland), $1-6,2000$.

[11] I. Chueshov and I. Lasiecka, Attractors for second-order evolution equations with a nonlinear damping, J. of Dyn. and Diff. Equations, 16 (2004), 469-512.

[12] I. Chueshov and I. Lasiecka, long-time behavior of second-order evolutions with nonlinear damping, Memoires of AMS, v. 195, 2008.

[13] I. Chueshov and I. Lasiecka, Generation of a Semigroup and Hidden Regularity in Nonlinear Subsonic Flow-Structure Interactions with Absorbing Boundary Conditions. Jour. Abstr. Differ. Equ. Appl. 3 (2012), 1-27. 
[14] I. Chueshov and I. Lasiecka, Von Karman Evolution Equations, Springer Verlag, 2010.

[15] I. Chueshov and I. Lasiecka, Well-posedness and long time behavior in nonlinear dissipative hyperbolic-like evolutions with critical exponents, preprint arXiv:1204.5864v1, April 2012.

[16] I. Chueshov, I. Lasiecka, and J.T. Webster, Evolution semigroups for supersonic flow-plate interactions, preprint arXiv:1205.7066v1, May 2012.

[17] I. Chueshov and A. Rezounenko, Global attractors for a class of retarded quasilinear partial differential equations, C. R. Acad. Sci. Paris, Ser. 1, 321 (1995), 607-612.

[18] P. Ciarlet and P. Rabier, Les Equations de Von Karman, Springer, 1980.

[19] O. Diekmann, S. van Gils, S. Lunel, and H.-O. Walther, Delay Equations. Springer, 1995.

[20] E. Dowell, Aeroelasticity of Plates and Shells, Nordhoff, Leyden, 1975.

[21] E. Dowell, A Modern Course in Aeroelasticity, Kluwer Academic Publishers, 2004.

[22] E. Dowell, Nonlinear Oscillations of a Fluttering Plate, I and II, AIAA J., 4, (1966) 1267-1275; and $5,(1967) 1857-1862$.

[23] D.H. Hodges, G.A. Pierce, Introduction to Structural Dynamics and Aeroelasticity, Cambridge Univ. Press, 2002.

[24] P.G. Geredeli, I. Lasiecka, and J.T. Webster, Smooth attractors of finite dimension for von Karman evolutions with nonlinear damping localized in a boundary layer, J. of Differential Equations, in press.

[25] T. Von Karman, Festigkeitsprobleme in Maschinenbau, Encyklopedie der Mathematischen Wissenschaften, Leipzig, 4 (1910), 348-352.

[26] A.K. Khanmmamedov, Global attractors for von Karman equations with non-linear dissipation, J. Math. Anal. Appl, 318 (2006), 92-101.

[27] E.A. Krasil'shchikova, The Thin Wing in a Compressible Flow, Nauka, Moscow, 1978, in Russian.

[28] O. Ladyzhenskaya, Attractors for Semigroups and Evolution Equations, Cambridge University Press, Cambridge, 1991.

[29] J. Lagnese, Boundary Stabilization of Thin Plates, SIAM, 1989.

[30] I. Lasiecka and J.T. Webster, Generation of bounded semigroups in nonlinear flow-structure interactions with boundary damping, Math. Methods in App. Sc., DOI: 10.1002/mma.1518, published online December, 2011

[31] I. Lasiecka and J.T. Webster, Long-time dynamics and control of subsonic flow-structure interactions, Proceedings of the 2012 American Control Conference, June 2012.

[32] Mixed problem for hyperbolic equation of second order, J. Math. Kyoto Univ., 13 (1973), 435-487. 
[33] M. Shubov, Solvability of reduced Possio integral equation in theoretical aeroelasticity. Adv. Differential Equations, 15 (2010), 801-828.

[34] R. Temam, Infinite Dimensional Dynamical Systems in Mechanics and Physics, Springer-Verlag, 1988.

[35] J. Wu, Theory and Applications of Partial Functional Differential Equations, Springer, 1996.

[36] J.T. Webster, Weak and strong solutions of a nonlinear subsonic flow-structure interaction: semigroup approach, Nonlinear Analysis, 74 (2011), 3123-3136. 